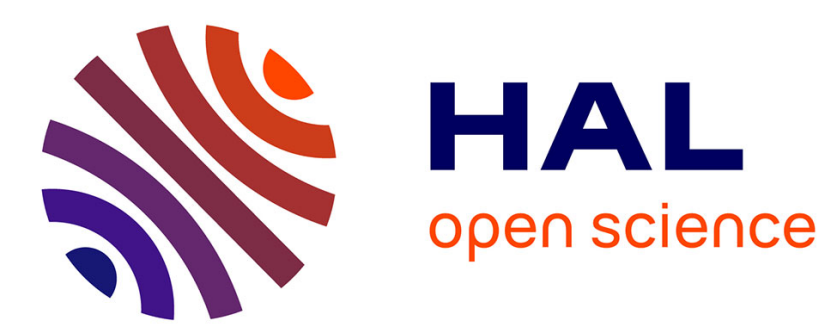

\title{
Effects of upstream perturbations on the solution of the laminar and fully turbulent boundary layer equations with pressure gradients
}

\author{
Jaime Vaquero, Nicolas Renard, Sébastien Deck
}

\section{To cite this version:}

Jaime Vaquero, Nicolas Renard, Sébastien Deck. Effects of upstream perturbations on the solution of the laminar and fully turbulent boundary layer equations with pressure gradients. Physics of Fluids, 2019, 31, pp.125103-1 - 125103-17. 10.1063/1.5125496 . hal-02431748

\author{
HAL Id: hal-02431748 \\ https://hal.science/hal-02431748
}

Submitted on 8 Jan 2020

HAL is a multi-disciplinary open access archive for the deposit and dissemination of scientific research documents, whether they are published or not. The documents may come from teaching and research institutions in France or abroad, or from public or private research centers.
L'archive ouverte pluridisciplinaire HAL, est destinée au dépôt et à la diffusion de documents scientifiques de niveau recherche, publiés ou non, émanant des établissements d'enseignement et de recherche français ou étrangers, des laboratoires publics ou privés. 


\section{Effects of upstream perturbations on the solution of the laminar and fully turbulent boundary layer equations with pressure gradients}

J. Vaquero, ${ }^{1}$ N. Renard, ${ }^{1,}$ a) and S. Deck ${ }^{1}$

ONERA The French Aerospace Lab

\section{Rue des Vertugadins, F-92190 Meudon, France}

(Dated: 8 November 2019)

The aim of this work is to contribute to the understanding of sensitivity of boundary layers to the upstream boundary condition and history effects for both laminar and fully turbulent states in equilibrium conditions as well as some non-equilibrium turbulent boundary layers. Solutions of the two-dimensional boundary layer equations are obtained numerically for this study together with the RANS (Reynolds-averaged Navier-Stokes) approach for turbulence modeling. The external pressure gradient is imposed through an evolution of the external velocity of the form $U_{e} \propto\left(x-x_{0}\right)^{m}$, and boundary layers are initialized from a profile giving a perturbed shape factor. It is found that laminar boundary layers require very long distances for convergence towards the non-disturbed profiles in terms of the initial boundary layer thickness $\left(\sim 10^{4} \delta_{\text {in }}\right)$ and that this distance is dependent on $m$. In turbulent boundary layers much shorter distances, although still large $\left(\sim 10^{2} \delta_{i n}\right)$, are observed and they are also dependent on $m$. The maximum adverse pressure gradient for which convergence to a reference solution is possible is also studied finding that there is no limit for attached laminar boundary layers, whereas turbulent boundary layers do not converge once they are out of equilibrium. The convergence distances in turbulent boundary layers are also studied in terms of the turnover length $\left(\delta U_{e}^{+}\right)$because it has been shown to be more appropriate to refer the convergence distance to this length rather than the boundary layer thickness. The values for convergence using this criterion are extended to pressure gradient boundary layers. Moreover an equivalent criterion is proposed and studied for laminar boundary layers based on the viscous characteristic time.

\footnotetext{
${ }^{a)}$ Electronic mail: nicolas.renard@onera.fr.
} 
Upstream perturbations effects on laminar and turbulent boundary layers

\section{INTRODUCTION}

When a boundary layer experiences a deviation from its reference or canonical state, the consequent effects persist during the development of the boundary layer and may diminish until they disappear farther downstream. These perturbations may be introduced either intentionally or accidentally but, in any case, significant effects on the development of the boundary layer are observed. Intentional introduction of perturbations is found in the triggering of laminar-to-turbulent transition, which is very often used for instance in experimental studies of turbulent boundary layers due to the limited physical space ${ }^{1}$. In numerical experiments of turbulent boundary layers, different methods to accelerate the appearance of the turbulent state are commonly considered to reduce the computational effort ${ }^{2}$. The impact of upstream perturbations on the downstream development of the boundary layer has been commonly referred to as history effects ${ }^{3-6}$. Such effects may modify substantially the development of the boundary layer and therefore it becomes essential to study whether the perturbed boundary layer will evolve towards a canonical state and, if it is the case, the way in which such evolution takes place. The aim of the present work is to study how upstream perturbations of the boundary layer impact the solution of the boundary layer equations in laminar and fully turbulent conditions in the presence of pressure gradients unchanged by the perturbation. It is important to point out that perturbations in this work are considered to apply directly to the upstream boundary layer profile, but other types of perturbations may exist as for instance in the external velocity distribution (and hence in the pressure gradient distribution) ${ }^{7}$. Due to the parabolic nature of the steady-state boundary layer equations, the upstream boundary conditions effects could be regarded as initial conditions effects whereas the external velocity distribution may be regarded as a boundary condition.

A canonical boundary layer can be considered when self-similarity is reached. This means that there is no evolution of the boundary layer profile in the streamwise direction when scaled with the proper parameters. Falkner and Skan ${ }^{8}$ showed that such a solution is possible in pressure-gradient laminar boundary layers as soon as the external velocity follows the law $U_{e} \propto x^{m}$, with a minimum value $m \approx-0.0904$ corresponding to zero friction coefficient (boundary layer separation) $)^{9,10}$. In the case of turbulent boundary layers, self-similarity in the whole profile is not possible (except in the case of the sink flow) because of the separation of scales between the inner and the outer layer. However, Clauser ${ }^{3}$ suggested that boundary layers could be obtained in near-equilibrium conditions if the ratio between the pressure gradient and the wall-shear forces is constant during 
the evolution of the boundary layer, provided a proper length scale is considered for the pressure gradient forces. Near-equilibrium conditions allow for a collapse of the velocity defect profile in the outer layer, which is much bigger than the inner layer, all the more so as the Reynolds number is important. In his later work, Clauser ${ }^{11}$ defined properly the previous ratio as $\beta=$ $\left(\delta_{1} / \tau_{w}\right) \mathrm{d} P_{e} / \mathrm{d} x$. In this definition $\tau_{w}=\mu(\partial\langle u\rangle / \partial y)_{y=0}$ is the mean wall-shear stress, $P_{e}$ is the external static pressure and $\delta_{1}$ the displacement thickness. The streamwise coordinate and velocity component are $x$ and $u$ respectively, $\mu$ is the dynamic viscosity, $y$ the wall-normal coordinate defined such that it vanishes at the wall and $v$ its corresponding velocity component, $\langle\bullet\rangle$ refers to the Reynolds average and the subscript ' $w$ ' refers to the wall. Clauser ${ }^{3}$ suggested that $\beta$ as well as the parameter $G$ should be invariant in the streamwise direction for near-equilibrium turbulent boundary layers. The latter variable is defined as

$$
G=\frac{\int_{0}^{\infty}\left(\frac{U_{e}-\langle u\rangle}{u_{\tau}}\right)^{2} \mathrm{~d} y}{\int_{0}^{\infty}\left(\frac{U_{e}-\langle u\rangle}{u_{\tau}}\right) \mathrm{d} y}=\frac{H-1}{H\left(\frac{C_{f}}{2}\right)^{\frac{1}{2}}} .
$$

where $C_{f}$ is the friction coefficient given by $C_{f} / 2=\tau_{w} /\left(\rho_{w} U_{e}^{2}\right)$ with $\rho$ the density, and $u_{\tau}$ is the friction velocity, defined such that $\tau_{w}=\rho_{w} u_{\tau}^{2}$, and $H=\delta_{1} / \theta$ is the shape factor defined as the ratio between the displacement and the momentum thicknesses $\delta_{1}$ and $\theta$ respectively. The upper limits of the integrals in equation (1) are taken as infinity because the boundary layer hypothesis are considered to apply. These hypothesis imply that the streamwise length scale is much greater than the wall-normal length scale and therefore $y \rightarrow \infty$ refers in fact to the edge of the boundary layer. This notation will be adopted from now on in the present work.

It is well-known that self-similarity is possible in laminar boundary layers in pressure gradient conditions when streamwise velocity profiles satisfy the Falkner-Skan equation ${ }^{8}$, and that such profiles are obtained for outer velocity distributions given by $U_{e} \propto x^{m}$. Not much work is devoted to the study of history effects in laminar boundary layers probably because of a larger importance of stability and laminar-to-turbulent transition studies. Gallo, Gnos, and Marvin ${ }^{12}$ studied hypersonic laminar boundary layers in both favorable and adverse pressure gradient conditions and showed that history effects were relevant especially in the adverse pressure gradient case and non-similar terms were not found to be very important in boundary layers with weak pressure gradients, in accordance with the results of Marvin and Sinclair ${ }^{13}$. Serrin ${ }^{14}$ and Peletier ${ }^{15}$ studied the asymptotic evolution of a laminar boundary layer from an arbitrary initial profile in favorable 
pressure gradient with an external velocity distribution $U_{e}(x)$ corresponding to that giving the solution of the Falkner-Skan equation. They showed that the boundary layer will always develop towards a solution of this equation. In adverse pressure gradient conditions, Chen and Libby 16 showed through linear theory that laminar boundary layers are spatially stable when developing from a slightly perturbed profile as long as the solution of the Falkner-Skan equation considered is that of positive friction coefficient ${ }^{17}$.

Near-equilibrium conditions for turbulent boundary layers are possible for an external velocity distribution akin to that giving self-similar solutions in laminar boundary layers as demonstrated by $\operatorname{Rotta}^{18}$, Mellor and Gibson ${ }^{19}$ and Townsend ${ }^{20}$ who obtained near-equilibrium conditions for $U_{e} \propto\left(x-x_{0}\right)^{m}$ where $x_{0}$ is a virtual origin. Rotta ${ }^{18}$ and Townsend ${ }^{20}$ also showed that selfpreservation in turbulent boundary layers is only satisfied for the whole boundary layer profile in the case of the sink flow in a smooth wall which is obtained for $m=-1$ and $x<x_{0}$ (a negative constant must be included for $\left.U_{e}(x)\right)$. In the present work, all the turbulent boundary layers are considered with $U_{e} \propto\left(x-x_{0}\right)^{m}$ and $x>x_{0}$ in a smooth wall and so the sink flow is not studied (which is a choice from the authors to limit the study to a coherent set of cases since sink flows and highly accelerated boundary layers are important research topics in themselves ${ }^{21,22}$ ). Contrary to self-similarity in laminar boundary layers, near-equilibrium is not possible for all the values of $m$ leading to attached flow in turbulent boundary layers. Head ${ }^{23}$ studied turbulent boundary layers in adverse pressure gradients with $U_{e} \propto\left(x-x_{0}\right)^{m}$ using a method based on integral quantities. He found that depending on the value of $m$, different initial conditions could either lead to a single equilibrium boundary layer, several states of equilibrium or non-equilibrium. Schofield ${ }^{24}$ revised the conclusions of Head studying turbulent boundary layers by means of the law of Perry and Schofield ${ }^{25}$ for the velocity defect which is valid for moderate and intense adverse pressure gradients. He found that turbulent boundary layers in adverse pressure gradient conditions such that $m<-0.23$ can exist in two different states of equilibrium: one with a large velocity defect (high $\beta$ and $H$ ) and another with a lower velocity defect (low $\beta$ and $H$ ). The latter case is the one considered in the present work for adverse-pressure-gradient turbulent boundary layers. This explains the lower values of the shape factor obtained by Clauser ${ }^{3}$ and Lee ${ }^{26}$ compared to the higher (close to separation) values of Stratford ${ }^{27}$, Mellor and Gibson ${ }^{19}$ or Kitsios et al. ${ }^{28}$ for the same value of $m$. Besides, Schofield also found that near-equilibrium is not possible when $m<-0.3$ which is a threshold in accordance with the results of $\operatorname{Head}^{23}$ and close to the value of $-1 / 3$ given by Townsend $^{20}$. These two conclusions are also observable in the work of Clauser ${ }^{3}$. 
Despite the numerous works studying zero-pressure-gradient turbulent boundary layers and the importance of the laminar-to-turbulent transition triggering either in experimental or numerical studies, not many works have detailed the evolution of the boundary layer from tripping conditions in order to ascertain the proper development of the boundary layer until recently ${ }^{29-31}$. Schlatter and Örlü ${ }^{32}$ carry out a comparison of different direct numerical simulations (DNS) data of zero-pressure-gradient turbulent boundary layers and they find important scatter in what should be canonical flows. In order to better understand the source of such disagreement, Schlatter and Örlï ${ }^{30}$ perform the same numerical simulation as in Schlatter and Örlü ${ }^{32}$ but under different tripping conditions and they compare them to the previous database. They show that the collapse between different quantities of the flow requires some development length after transition takes place. An interesting finding in their work is that agreement between different simulations happens for $R e_{\theta}>2000$ if tripping is made at $\operatorname{Re}_{\theta}<300\left(\operatorname{Re}_{\theta}=U_{\infty} \theta / v\right.$, where $U_{\infty}$ is the freestream velocity, $\theta$ is the momentum thickness and $v=\mu / \rho$ the kinematic viscosity) showing that convergence requires a quite long range of Reynolds numbers which may be an issue for either experimental or numerical studies. In addition, their results suggest that the outer layer requires longer distances than the inner layer to achieve flow history independence, in accordance with the results of Clauser ${ }^{11}$, Marusic et al. ${ }^{31}$ and Sanmiguel Vila et al. ${ }^{33}$ for instance.

Marusic et al. ${ }^{31}$ also study the evolution of zero-pressure-gradient turbulent boundary layers with different tripping conditions but experimentally, reaching thus higher Reynolds numbers (up to $\left.R e_{\theta} \sim 30000\right)$ than direct numerical simulations ${ }^{30,34,35}$. Tripping rods with different geometries are used to trigger the transition to turbulence. For the different tripping conditions convergence is always observed, although the distances to reach it, which are in the range of $130 \delta$ to $200 \delta$ ( $\delta$ is the boundary layer thickness), are dependent on the tripping device, a result observed by Sanmiguel Vila et al. ${ }^{33}$ as well. The main discrepancies between their different experiments are found in the outer layer of boundary layers, where more energetic structures develop when bigger rods are used, in accordance with the results of Sanmiguel Vila et al. ${ }^{33}$.

Some recent works have been devoted to the identification of well-behaved turbulent boundary layers. This refers to boundary layers that reach a state free from history effects and tripping or inflow conditions. Chauhan, Monkewitz, and Nagib ${ }^{36}$ study zero-pressure-gradient turbulent boundary layers and show that the use of the shape factor $H$, the wake factor $\Pi$, the ratio $x / \delta$ (where $x$ is the distance from the virtual origin of the turbulent boundary layer in their work) and the time scale ratio $\Omega$ between the mean flow and the turbulence, may be good criteria for 
the identification of canonical boundary layers. They also provide advises for well-documented experimental studies that are very useful for determining whether a given boundary layer reaches well-behaved conditions. Sanmiguel Vila et al. ${ }^{33}$ apply the diagnostic-plot method ${ }^{37}$ for zeropressure-gradient turbulent boundary layers, which has the advantage of not relying on difficult measurements (for experimental studies) like the friction velocity or other quantities related to the inner layer, but only on measurements of the streamwise mean velocity and turbulence intensity in the outer layer at some arbitrary positions. Vinuesa et al. ${ }^{6}$ suggest three different criteria to identify well-behaved turbulent boundary layers in adverse pressure gradient conditions depending on the history of the flow. The first one is suitable for constant- $\beta$ flows and relies on empirical curves of $C_{f}$ or $H$. The two other criteria are suggested for general evolutions of $\beta$ as a function of $\operatorname{Re}_{\theta}$. From these two criteria, the first one is based on empirical $C_{f}$ curves and the second one is based on the diagnostic plot modified with $H$. However the latter criterion has the disadvantage of requiring profile measurements at some defined positions as well as the determination of the boundary layer thickness and the computation of the shape factor, contrary to that of Sanmiguel Vila et al. ${ }^{33}$ for zero-pressure-gradient conditions.

Although global quantities like the friction coefficient or the shape factor may be considered as good indicators of the state of the boundary layer ${ }^{36}$, more information about the impact of history effects may be obtained from other quantities. In particular, the mean streamwise velocity profiles as well as the profiles of the streamwise turbulence intensity evidence a faster convergence of the inner layer with respect to the outer layer ${ }^{11,30,31,33}$. Some discrepancy has been observed regarding the profiles of the Reynolds shear stress and the wall-normal Reynolds normal stress as well as for higher order statistics. Castillo and Johansson ${ }^{38}$ and Seo et al. ${ }^{39}$ find a lower convergence speed for the wall-normal Reynolds normal stress and the Reynolds shear stress with respect to the mean streamwise velocity profile. However, this conclusion is not supported by the more recent work of Schlatter and Örlü ${ }^{30}$. Moreover, Marusic et al. ${ }^{31}$ observe that the convergence speed is not dependent on the order of the statistics of the streamwise velocity, contrary to Smits and Wood ${ }^{7}$, and the results from Sanmiguel Vila et al. ${ }^{33}$ do not seem to show a difference in the convergence speeds between the streamwise turbulence intensity and the streamwise mean velocity profiles either.

Despite the works focusing on the identification of well-behaved boundary layers, the definition of a proper convergence distance from upstream conditions in boundary layers is still an open question $^{5,31}$. In the early works some discrepancies have already been obtained. For instance, 
according to Herring and Norbury ${ }^{40}$, Jones and Launder ${ }^{41}$ or Inman and Bradshaw ${ }^{42}$, distances of about 30 boundary layer thicknesses are required to obtain equilibrium conditions in favourablepressure-gradient turbulent boundary layers. However, Clauser ${ }^{11}$ finds that, for tripping zeropressure-gradient boundary layers, longer distances are required to obtain a canonical boundary layer and that these distances, which are of about a hundred boundary layer thicknesses, are mainly due to the recovery of the outer layer, similarly to Schlatter and Örlü ${ }^{30}$ or Marusic et al. ${ }^{31}$. Bobke et al. ${ }^{5}$ compare several turbulent boundary layers with different flow histories in adverse pressure gradients, showing the importance of such effects when comparing at matched non-dimensional parameters $\left(\beta\right.$ and $\left.R e_{\tau}=\delta u_{\tau} / v\right)$. A boundary layer subjected to a sudden pressure gradient such that $\beta$ increases from zero to a given value shows less impact of the pressure gradient on its mean velocity and streamwise turbulence intensity profiles than a boundary layer subjected to an approximately constant $\beta$ at about the same value than that of the first boundary layer. In other words, this shows that history effects are essential in the impact of the pressure gradient on the boundary layer. Bobke et al. also find that a boundary layer developing with a smooth evolution of $\beta(x)$ collapses to a constant- $\beta$ boundary layer of similar value of $\beta$ to those of the first boundary layer, $\beta(x)$, at a distance of about $7 \bar{\delta}_{99}$, being $\bar{\delta}_{99}$ the mean boundary layer thickness defined such that $\langle u\rangle=0.99 U_{e}$. Simens et al. ${ }^{43}$ rather suggest the use of the turnover length $L_{T O}$ to characterize the convergence from history effects. It is defined as the distance traveled by a large eddy of size $O(\delta)$ during a turnover time $\left(\delta / u_{\tau}\right)$ convected by a velocity of about $U_{e}$. Based on this idea, Sillero et al. ${ }^{34}$ suggest the variable $\tilde{x}_{T}$

$$
\tilde{x}_{T}=\int_{x^{*}}^{x} \frac{\mathrm{d} x}{L_{T O}}=\int_{x^{*}}^{x} \frac{\mathrm{d} x}{\delta U_{e}^{+}}
$$

(where $U_{e}^{+}=U_{e} / u_{\tau}$ and $x^{*}$ denotes the beginning of the streamwise extent considered which may be the inflow position or a location related to transition to turbulence) also used by Sillero, Jiménez, and Moser ${ }^{35}$ who find convergence distances of the order of $\tilde{x}_{T} \approx 1$ for variables related to the small-scale structures and $\tilde{x}_{T} \approx 4$ for those related to the large ones. They apply this criterion to different Reynolds-number computations obtaining similar results and showing thereby its low Reynolds-number dependence, contrary to convergence distances based on the boundary layer thickness.

Equilibrium laminar boundary layers are easy to compute thanks to their self-similar character as well as the absence of need for a closure for the Reynolds shear stress. Different approaches can be found for near-equilibrium turbulent boundary layers, aside from experimental works and 
turbulence-resolving numerical simulations. For instance, in the work of $\mathrm{Head}^{23}$, solutions are obtained by means of a method based on global quantities and Schofield ${ }^{24}$ uses a law of the mean streamwise velocity given by Perry and Schofield ${ }^{25}$ which is valid for moderate to strong adverse pressure gradients. According to Smits and $\mathrm{Wood}^{7}$, the only analytical method to analyze perturbed turbulent boundary layers is the rapid-distorsion theory but they add that, even if the initial response can be described, it is not the case for the relaxation process. More recently, Perry, Marušić, and $\mathrm{Li}^{44}$ have proposed a turbulence closure based on log-layer and wake laws and mean continuity and momentum differential and integral equations for arbitrary pressure gradients. In addition, an extension of the attached eddy hypothesis ${ }^{20}$ is included by Perry and Marusic ${ }^{45}$. This closure has been used by Marusic and Perry ${ }^{46}$ and Perry, Marusic, and Jones ${ }^{47}$ in non-equilibrium adverse-pressure-gradient turbulent boundary layers and by the latter in sink flows approaching equilibrium as well. Marusic et al. ${ }^{31}$ have also applied this closure to their zero-pressure-gradient boundary layers showing not only good agreement with experimental results but also convergence from all the different initial conditions of the boundary layers towards what should be a canonical boundary layer.

The tool used in the present work solves the steady boundary layer equations. This means that the only hypothesis is to assume that these apply for all points of the computational domain. The tool stops the simulation automatically whenever boundary layer separation is detected. Assuming the Boussinesq's hypothesis for the closure of the Reynolds shear stress, several models are tested in order to reduce the uncertainty from turbulence modeling. Thanks to the computation of the two-dimensional boundary layer equations together with the RANS (Reynolds-averaged NavierStokes) approach, solutions of the simulations are obtained extremely fast compared to turbulence resolving approaches, which allows not only to perform several simulations as it is done in this work, but also to reach high Reynolds numbers, which is of special interest for turbulent boundary layers due to the high computational effort of other methods involving less or no turbulence modeling.

In the present work, the effects of upstream perturbations in laminar and high-Reynolds-number turbulent boundary layers are investigated for zero pressure gradient conditions as well as selfsimilar laminar boundary layers and near-equilibrium turbulent boundary layers both in favorableand adverse-pressure-gradient conditions. Such conditions are obtained thanks to external velocity distributions given by $U_{e} \propto\left(x-x_{0}\right)^{m}$ in all cases and the mean flow is considered steady, twodimensional and incompressible. There are several questions to which we try to bring a clearer 
Upstream perturbations effects on laminar and turbulent boundary layers

insight by means of the present study:

1. What is the extension of the region affected by upstream effects in the boundary layer?

2. Does this distance depend on the laminar or turbulent character of the boundary layer?

3. How does the value of the external pressure gradient influence the convergence of the boundary layer?

4. Is there always a convergence for any value of the pressure gradient?

For that purpose, the perturbations are introduced in the same way for all the cases considered and therefore the study allows to analyze different boundary layers under the same type of perturbations. The present analysis is complementary to other works which focus on boundary layers of one kind experiencing different types of perturbations as for instance in the work of Schlatter and Örlü ${ }^{30}$ or Marusic et al. ${ }^{31}$.

This paper is organized as follows. In §II B the procedure used for the set-up of the simulations and the generation of the perturbed and reference boundary layers is explained. In section $\S$ III the convergence distances for laminar and turbulent boundary layers based on the boundary layer thickness are studied together with the maximum intensity of the adverse pressure gradient for convergence. In section $\S \mathrm{IV}$, the convergence length $\tilde{x}_{T}$ in pressure gradient conditions is studied and an equivalent convergence length based on the viscous characteristic time for laminar boundary layers is presented. Finally, section $\S \mathrm{V}$ summarizes the main results of this work.

\section{PROCEDURE FOR THE BOUNDARY LAYERS ANALYSIS}

\section{A. Numerical tool presentation}

Numerical simulations of both laminar and high-Reynolds-number turbulent boundary layers are performed using the ONERA boundary-layer code CLICET ${ }^{48}$. This code solves the steady two-dimensional compressible boundary layer equations thanks to a finite volume method and gives the possibility to choose between several turbulence models for the RANS approach in turbulent boundary layers. A brief description of the equations solved is given in appendix A and a numerical experiment has also been carried out to validate the use of boundary layer equations in strong adverse-pressure-gradient conditions compared to the full Navier-Stokes equations. 
The solver has been used in studies such as that of Aupoix and Spalart ${ }^{49}$ for the wall roughness modeling or by Renard and Deck ${ }^{50}$ in their study of the mean skin friction coefficient decomposition in zero-pressure-gradient turbulent boundary layers at high Reynolds numbers. For the highReynolds-number turbulent boundary layers of this work, the computations start with a Reynolds number based on the momentum thickness, $R e_{\theta}$, between $1.0 \times 10^{4}$ and $1.4 \times 10^{4}$, and reach values between $7.3 \times 10^{5}$ and $1.5 \times 10^{6}$, which are much greater than those reached by DNS and Wall-Resolved Large-Eddy Simulation (WRLES) ${ }^{51}$. Although such values of Reynolds number may be reached by Wall-Modeled Large-Eddy Simulation (WMLES) (see for instance the work of Inoue et al. ${ }^{52}$ or Deck et al. ${ }^{53}$ ), the huge computational effort still required for this approach justifies the interest of the present tool. The solver uses an automatic mesh refinement in order to increase the number of points where needed. Besides, several criteria are also considered to validate the quality of the mesh, such as the position of the first point away from the wall within the inner layer (for turbulent boundary layers).

\section{B. Generation of the reference and perturbed computations}

In the present work, the evolution of the perturbed boundary layer from an initial profile is studied by means of the shape factor $H$ which is compared to that of a reference boundary layer developing under the same conditions as the perturbed boundary layer without introducing the perturbation. The latter is generated as detailed in the next paragraph at the inlet profile. Both boundary layers (the reference one and the perturbed one) are subjected to an external pressure gradient resulting from an external velocity law (boundary condition) of the type $U_{e} \propto\left(x-x_{0}\right)^{m}$ unchanged by the perturbation introduced. This law allows to obtain self-similar conditions in laminar boundary layers (since such a distribution provides a solution of the Falkner-Skan equation ${ }^{8}$ ) and near-equilibrium conditions in turbulent boundary layers as long as $m>-0.3^{24}$. All the computations in this work have been performed at low external Mach number $\left(M_{e} \leq 0.2\right)$ in order to avoid compressibility effects and it has been confirmed by looking at the relative variation of density and molecular viscosity across the boundary layer in the case of highest Mach number, showing variations well below $1 \%$.

It is important to remind that the pressure gradient has an impact on $H$, and so does the Reynolds number in the case of turbulent boundary layers. For both laminar and turbulent boundary layers, an adverse pressure gradient will tend to increase the shape factor ${ }^{8,54-56}$. In the work of Monty, 
Harun, and Marusic ${ }^{56}$, the effects of the Reynolds number and the pressure gradient on the shape factor of turbulent boundary layers are assessed individually. According to them, for low and moderate adverse pressure gradients at constant $\beta$, the evolution of $H$ is similar to that in zeropressure-gradient conditions i.e. it decreases with the Reynolds number. The values of $H$ are however modified due to the pressure gradient (greater values for adverse pressure gradients). It is also possible to see such a trend in the work of Bobke et al. ${ }^{5}$. In the case of strong adverse pressure gradients, a general trend is less evident. Indeed, according to the results of Skåre and $\operatorname{Krogstad}^{54}$, the shape factor at constant $\beta$ decreases with the Reynolds number, whereas the DNS results of Kitsios et al. ${ }^{28}$ show rather an increase of the shape factor. Other studies such as that from Nagib and Chauhan ${ }^{57}$ have analysed the impact of the pressure gradient on the coefficients of the log-law, which will modify the mean velocity profile and therefore the shape factor. In appendix A, a validation of the shape factor evolution obtained from the present solver CLICET in adverse-pressure-gradient conditions is presented against experimental data.

The procedure followed for the generation of the reference and perturbed boundary layers is now detailed. The boundary layer is disturbed by a perturbation of the upstream boundary condition i.e. of the initial boundary layer profile of mean streamwise velocity, as well as mean turbulent kinetic energy and mean turbulent-kinetic-energy dissipation if turbulence simulations are performed. We consider a first boundary layer BL1 developing over a flat plate from a stagnation point and an external velocity distribution given by $U_{e} \propto\left(x-x_{0}\right)^{m}$ (figure 1 (top left)). At the streamwise position $x_{i n}$ the boundary layer BL1 has a shape factor $H_{r e f}$ and a momentum thickness $\theta_{i n}$. A second boundary layer BL2 is computed again from a stagnation-point-like inlet condition (uniform flow) under the same kind of external velocity law, but with a different exponent $m_{\text {per }} \neq m$ (figure 1 (bottom left)). Due to the different external velocity laws, the second boundary layer (BL2) will reach the same momentum thickness $\theta_{i n}$ as the first boundary layer (BL1) at a different streamwise position $\left(x_{2} \neq x_{i n}\right)$ and it will also have a different shape factor $\left(H_{\text {per }} \neq H_{\text {ref }}\right)$.

Two more computations, denoted BL3 and BL4 respectively, are then performed as presented in figure 1. These are the computations used for the analysis in the present work (BL1 and BL2 are only precursor simulations to give the proper inlet conditions for BL3 and BL4), which is why they are also denoted $\mathrm{BL}_{r e f}$ and $\mathrm{BL}_{\text {per }}$ respectively in figure 1 . Both computations start at $x_{i n}$ (and not from a stagnation point) with the same external velocity distribution as in BL1 $U_{e} \propto\left(x-x_{0}\right)^{m}$ and with a longer streamwise extent than BL1 and BL2. The first one of the two new computations 
(BL3) starts with the same profile as BL1 at $x_{i n}$, so it has an initial shape factor $H_{r e f}$ and an initial momentum thickness $\theta_{i n}$. In the case of BL4, the initial profile is given by that of BL2 at the streamwise position giving $\theta_{i n}$, which is $x_{2}$ (figure 1 (bottom)). Before using this profile as the upstream boundary condition for BL4, some modifications are done so that the external velocity at $x_{\text {in }}$ corresponds to that of the external velocity law at that position. This is done in order to satisfy the boundary condition at the edge of the boundary layer because the external velocity of the extracted profile of BL2 is different to that of BL1 since different external velocity laws are used.

In practice, an additional modification is also required in the described procedure. This modification is again applied to the inlet boundary layer profile of BL4 in order to have exactly the same momentum thickness $\left(\theta_{i n}\right)$ than BL1 and BL3. Since the computational domain is discrete, the position $x_{2}$ for BL2 will not exactly give $\theta_{\text {in }}$ (in general). The actual point used for $x_{2}$ is the closest to that giving $\theta_{\text {in }}$ in order to minimize the differences between $\theta_{\text {in }}$ of BL1 and that obtained numerically for BL2 (so $\theta_{2} \approx \theta_{i n}$ ). Then the profile of BL4 (extracted from BL2) is modified according to (3) and the computations of BL3 and BL4 are carried out, where BL3 is the reference boundary layer and BL4 the perturbed boundary layer. Moreover, since for both of them the inlet external velocity and momentum thickness are the same, the inlet $\operatorname{Re}_{\theta}$ is unchanged.

$$
y_{4}=y_{2} \frac{\theta_{i n}}{\theta_{2}}, \quad\langle u\rangle_{4}=U_{e, i n} \frac{\langle u\rangle_{2}}{U_{e, 2}} .
$$

In (3), $y$ is the wall normal coordinate and this equation corresponds to the only adjustment performed for laminar boundary layer computations, where the mean streamwise velocity is the total streamwise velocity.

Regarding the turbulent boundary layer computations, additional adjustments are required for the profiles of the turbulent kinetic energy $k$ and the turbulent-kinetic-energy dissipation $\varepsilon$. The friction velocity $u_{\tau}$ is considered the velocity scale for the Reynolds shear stress $-\left\langle u^{\prime} v^{\prime}\right\rangle$ and the profile of $k$ is modified taking $k \propto u_{\tau}^{254,58,59}$. The friction velocities $u_{\tau, 2}$ and $u_{\tau, 4}$ for the extracted profile of BL2 and the inlet profile of BL4 respectively are obtained from the mean velocity profiles once the adjustment from equation (3) is made. With these friction velocities the inlet profile of turbulent kinetic energy for BL4 $\left(k_{4}\right)$ may be obtained from equation (4) where the adjustment for $\varepsilon$ is also given. This one requires an additional change which implies the eddy viscosity $v_{t}$. Since the profile of $\varepsilon$ is only needed for the two-equation turbulence models, it is considered $v_{t}=C_{\mu} k^{2} / \varepsilon$, where $C_{\mu}$ is a constant used in the $k-\varepsilon$ turbulence model $^{60,61}$. It is then possible 


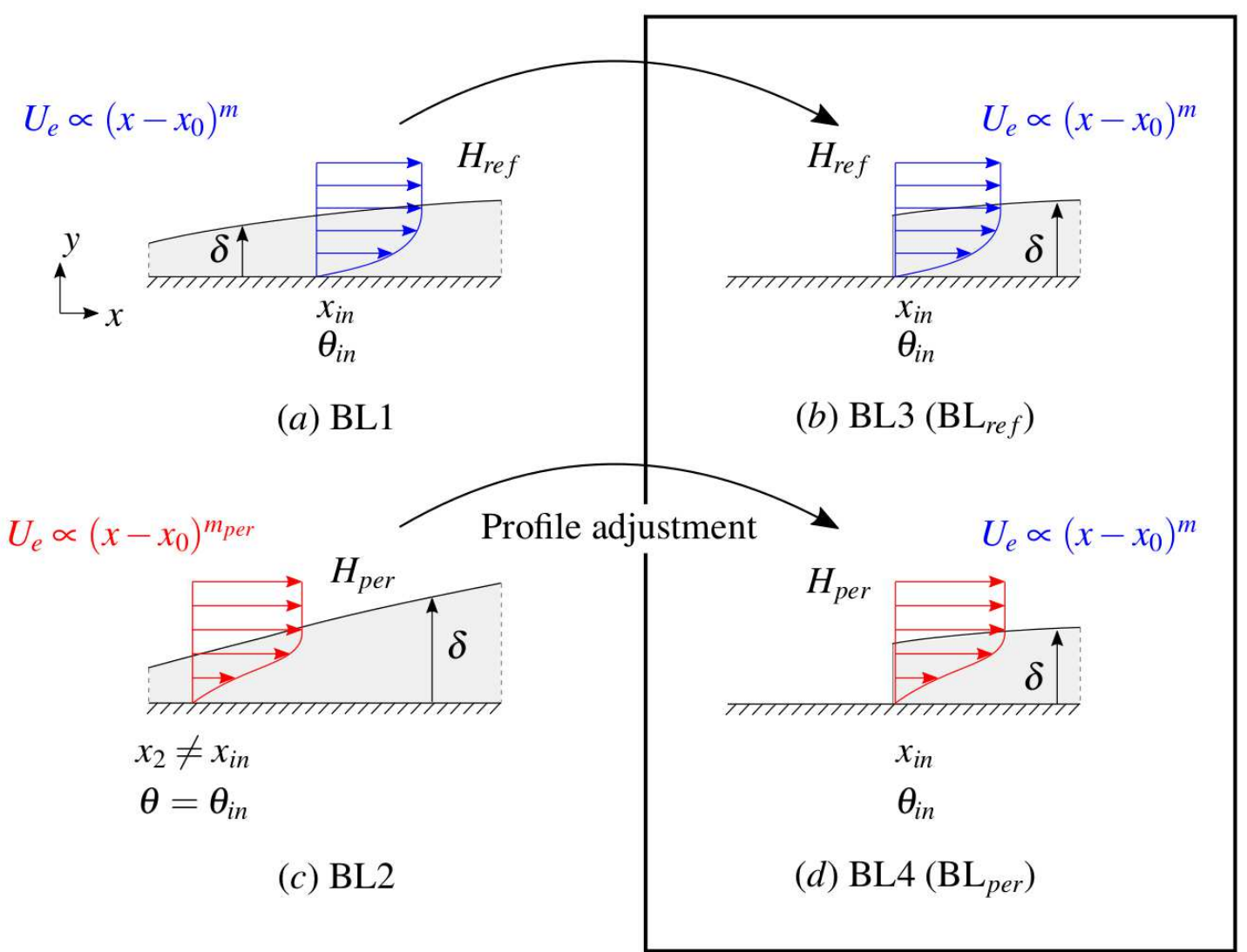

FIG. 1. Schematic of the perturbation introduction. Grey regions inside the boundary layer are used to show the different inlets of the computational domains. (a): boundary-layer computation from stagnationpoint-like inlet conditions with reference external flow conditions (BL1). (b): boundary-layer computation with reference external flow conditions and inlet conditions from BL1 profile at $x_{i n}$ (BL3). (c): boundarylayer computation from stagnation-point-like inlet conditions with different external flow conditions than the reference, $m_{\text {per }} \neq m$ (BL2). (d): boundary-layer computation with reference external flow conditions and inlet conditions from BL2 profile at $x_{2}$ (BL4).

to obtain the profile of $\varepsilon_{4}$ to be introduced from equation (4), where the eddy viscosity profile is recomputed from the Boussinesq's hypothesis by means of the Reynolds shear stress and the mean streamwise velocity profiles.

$$
\frac{k_{4}(y)}{k_{2}(y)}=\frac{u_{\tau, 4}^{2}}{u_{\tau, 2}^{2}}, \quad \frac{\varepsilon_{4}(y)}{\varepsilon_{2}(y)}=\frac{k_{4}(y)^{2}}{k_{2}(y)^{2}} \frac{v_{t, 2}(y)}{v_{t, 4}(y)}
$$

Although Schlatter and Örlü ${ }^{30}$ indicate that the analysis of history effects by means of the shape factor or the mean skin-friction coefficient is not sufficient and that profiles of mean velocity and higher order statistics should also be exploited, the perturbed-case shape factor is studied in the 


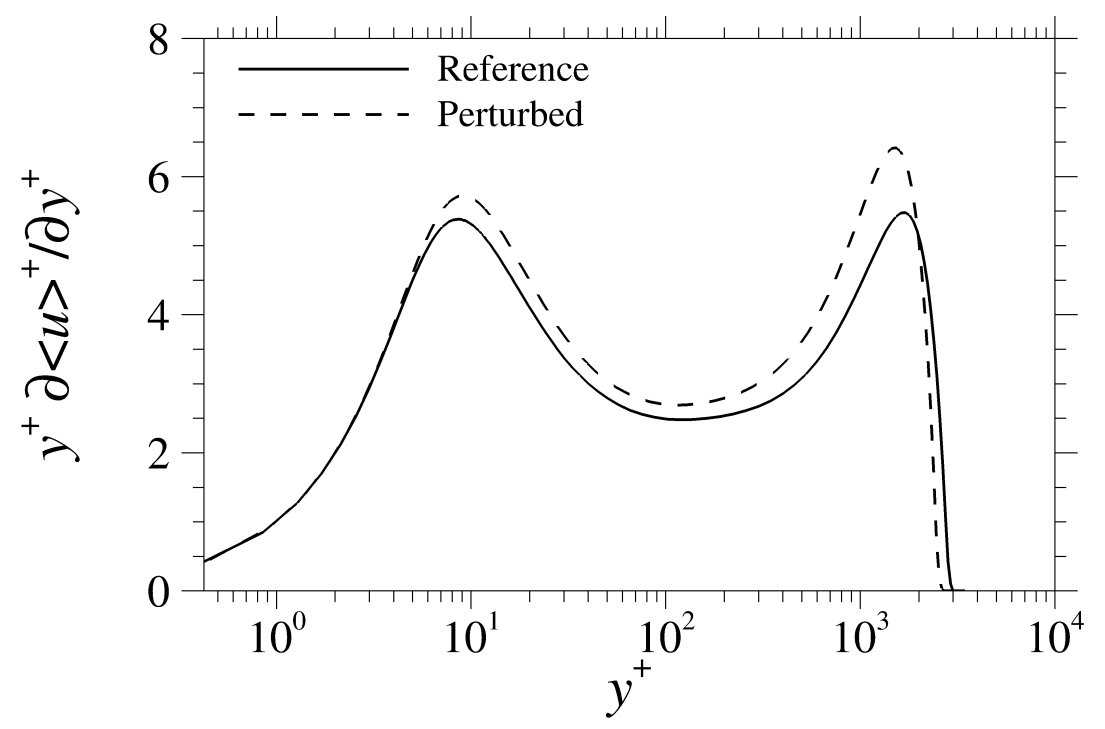

FIG. 2. Comparison of $y^{+} \partial\langle u\rangle^{+} / \partial y^{+}$for a reference and a perturbed mean streamwise velocity profile at $x_{\text {in }}$ for the zero-pressure-gradient turbulent boundary layer using the turbulence model of Michel, Quémard, and Durant ${ }^{62}$.

present work with respect to its (small) initial perturbation magnitude which gives a more detailed insight of the shape factor evolution. Furthermore, the evolutions of $u_{r m s}, v_{r m s}$ and $-\left\langle u^{\prime} v^{\prime}\right\rangle$ (where the subscript ' $r m s$ ' refers to the root-mean square value and $u_{i}^{\prime}=u_{i}-\left\langle u_{i}\right\rangle$ denotes the $i^{\text {th }}$ component of the fluctuating velocity) have not given any additional information to the main conclusions of the present study.

Marusic et al. ${ }^{31}$ find convergence for all the different tripping conditions studied in a zeropressure-gradient turbulent boundary layer, but the distances required for convergence are dependent on the way tripping is done as in the experiments of Klebanoff and Diehl ${ }^{63}$ and Sanmiguel Vila et al. ${ }^{33}$. For that reason, in this work the magnitude of the perturbation in the shape factor has been chosen sufficiently small so that the evolution of the perturbation downstream is not dependent on its initial amplitude $\Delta H_{\text {in }}=\left(H_{p e r}-H_{r e f}\right)_{\text {in }}$. In particular $\Delta H_{\text {in }}$ is in general around $1 \%$ or below of the inlet reference shape factor. In addition, all the boundary layers are perturbed in the same way, so that the analysis is made for boundary layers under the same kind of perturbation. Figure 2 shows an example of the difference between a reference profile and a perturbed profile at the inlet location $x_{i n}$ for a turbulent boundary layer computation. Instead of the mean velocity profile, its pre-multiplied wall-normal gradient is chosen so that small differences are highlighted. The superscript ' + ' means variables non-dimensionalized by wall units (the length scale is $v / u_{\tau}$ and 
the velocity scale $u_{\tau}$ ). In figure 3 the streamwise evolution of $\Delta H / \Delta H_{\text {in }}$ (with $\Delta H=H_{p e r}-H_{r e f}$ ) is presented for zero-pressure-gradient laminar and turbulent boundary layers. In this figure $\delta_{i n}$ is the boundary layer thickness at the inlet and the boundary layer thickness is defined as the wall normal position in which $\langle u\rangle=0.995 U_{e}$ and the values of $m$ are those used to generate the initial shape factor perturbation as described previously. In the case of turbulent boundary layers simulations, turbulence models of different nature are used thereby reducing the incertitude that turbulence modeling may incur in the conclusions drawn: the algebraic model of Michel, Quémard, and Durant $^{62}$, the one-equation model of Spalart and Allmaras ${ }^{64}$ and the two-equations $k-\varepsilon$ model in its version of Jones and Launder ${ }^{41}$. Besides, for the turbulent computations the version of the two-equation $k-\omega$ SST model of Menter ${ }^{65}$ has also been tested showing very similar results to the Jones and Launder ${ }^{41}$ model, but it is not presented for clarity in the results. The considered models are representative of the common best practice in turbulence modeling of industrial flows with pressure gradients. As observed in these figures, the use of small initial shape-factor differences allows to find evolutions of the perturbed-case shape factor that are independent of this initial value. Quite high values of the initial perturbation for the laminar boundary layer still show the same behavior.

The use of small perturbations could recall the classical stability analysis of boundary layers. However some differences exist between such studies and the present one. Firstly, stability analysis is most often devoted to the study of laminar-to-turbulent transition, which is not the objective in this work since computations are made for either fully laminar or fully turbulent boundary layers. Secondly, one may believe that stability analysis could be interesting for the case of fully laminar boundary layers. Even if this is true, stability is usually related to the temporal evolution of perturbations, which is not the case in this study since steady boundary layer equations are considered and the upstream perturbation is evolving in space (in the streamwise direction) rather than in time, taking advantage of the parabolic nature of the steady boundary layer equations. The boundary layer belonging to the convective dynamics group of stability analysis, perturbations are convected downstream but a temporal evolution would still be required (for instance by taking a convective derivative instead of the temporal one). However, this is not the usual procedure in stability analysis. Although it is still possible to perform stability analysis in these conditions, that would require the development and validation of a proper tool, not necessarily useful for other studies than the one presented. Moreover, the present tool allows for fast reliable results since the whole boundary layer equations are accurately solved (to the precision of the numerical schemes 

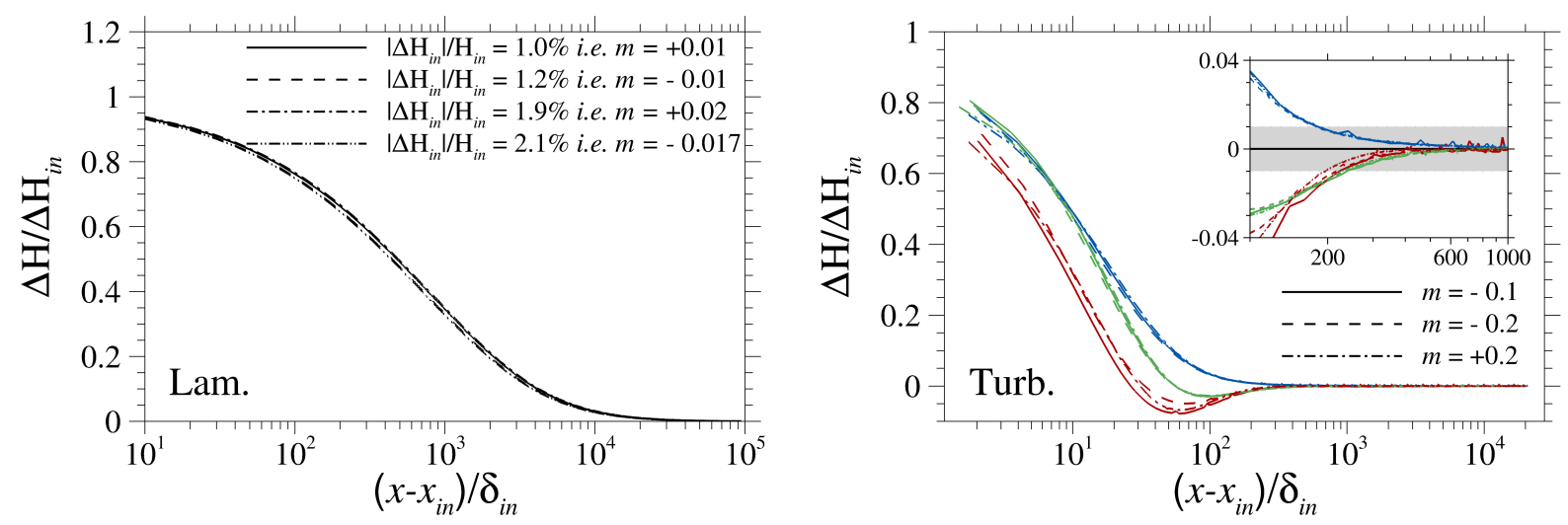

FIG. 3. Streamwise evolution of the shape factor difference in zero-pressure-gradient laminar (left hand side) and turbulent (right hand side) boundary layers for different initial shape-factor perturbations. Different turbulence models are used in (right hand side): Michel, Quémard, and Durant ${ }^{62}$ (blue), SpalartAllmaras (green) and $k-\varepsilon$ from Jones and Launder ${ }^{41}$ (red). The different line patterns correspond to different values of the initial shape-factor difference $\Delta H_{i n}$. The grey area in (right hand side) represents $1 \%$ difference. The values of $m$ shown in figures corresponds to those used to extract the boundary layer profile according to the procedure described in section II B. The range of Reynolds number for the turbulent simulations (right hand side) is $\operatorname{Re}_{\theta}$ from $8 \times 10^{3}$ to $10^{6}$.

used) and thus non-linear effects are also considered. Conversely, linear stability analysis typically involves modifications of the RANS turbulence models in order to make them differentiable, which may be an important issue for some models.

\section{Results validation}

The use of an external velocity given by a power $m$ of the streamwise coordinate gives selfsimilarity and near-equilibrium conditions in laminar and turbulent boundary layers respectively. That these conditions are satisfied can be verified thanks to some non-dimensional parameters presented by Clauser ${ }^{3,11}$. In particular, for laminar boundary layers the pressure gradient parameter $\beta$ and the shape factor $H$ should be streamwise independent, whereas for turbulent boundary layers, the parameter $G$ proposed by Clauser $^{3}$ (and given in equation (1)) replaces the shape factor as a near-equilibrium criterion. Indeed, Clauser defines this parameter similarly to the shape factor and based on the outer boundary layer self-similarity for the defect velocity scaled with the friction velocity (see equation (1)). 
The pressure-gradient parameters $\beta$ and $G$ are represented in figures 4 and 5 for the adversepressure-gradient turbulent boundary layer computations. For laminar boundary layers (not shown) $\beta$ and $H$ are independent of $x$ in most of the streamwise domain for all the simulations and the same happens for $\beta$ and $G$ in the favorable- and zero-pressure-gradient turbulent boundary layers for all models studied. One can observe that the above-mentioned near-equilibrium conditions are not satisfied for all the cases presented for turbulent boundary layers. In particular, near-equilibrium is obtained for $m=-0.15, m=-0.23$ and $m=-0.28$, but not for $m=-0.32$ and $m=-0.4$. This is in accordance with previous conclusions such as those from Clauser ${ }^{3}$, Head $^{23}$ or Schofield ${ }^{24}$ from which equilibrium is not possible for $m<-0.3$. Nevertheless, for the sake of generality in the present study, boundary layers out of equilibrium have also been considered, since there are many applications in which non-equilibrium boundary layers are found. It is also worth noticing how the near-equilibrium is reached farther downstream for the case $m=-0.28$ than for $m=-0.15$ and $m=-0.23$, in accordance with Piquet ${ }^{66}$ who indicates that the convergence to equilibrium conditions is slower as the intensity of the adverse pressure gradient increases. Besides, it is worth noticing the low values of $\beta$ for the near-equilibrium computations compared to those from other works using similar values of $m$ as for instance that of Mellor and Gibson ${ }^{19}$ or Kitsios et al. ${ }^{28}$. As mentioned in the introduction, this is due to the existence of two near-equilibrium states for $m<-0.23^{24}$. The computations in the present work belong to the state more distant to separation (lower $\beta$ and $H$ ) when $m<-0.23$.

Simens et al. ${ }^{43}$ suggest the turnover length $L_{T O}=U_{e}^{+} \delta$ as a more appropriate scale to measure the relaxation distance for the inflow conditions in turbulent boundary layers than the boundary layer thickness. It represents the distance traveled by eddies convected at the free-stream velocity during a turnover time $\delta / u_{\tau}$. Physically it seems a quite appropriate variable, since in turbulent boundary layers disturbances are forgotten more slowly in the outer layer ${ }^{11,20,30,31}$. The mean momentum equation very near the wall, which is (neglecting convective and Reynolds stress terms)

$$
\frac{\partial}{\partial y}\left(\mu \frac{\partial\langle u\rangle}{\partial y}\right)=\frac{\mathrm{d} P_{e}}{\mathrm{~d} x}
$$

shows that the inner layer responds faster to pressure gradient effects ${ }^{7}$. These effects are studied in the present work and therefore the use of the turnover length scale seems very appropriate.

Based on the turnover length, Sillero et al. ${ }^{34}$ suggest a non-dimensional turnover distance given by equation (2) and in a later work, Sillero, Jiménez, and Moser ${ }^{35}$ obtain that inflow effects are forgotten in a zero-pressure-gradient turbulent boundary layer at $\tilde{x}_{T} \approx 1$ for parameters related to 

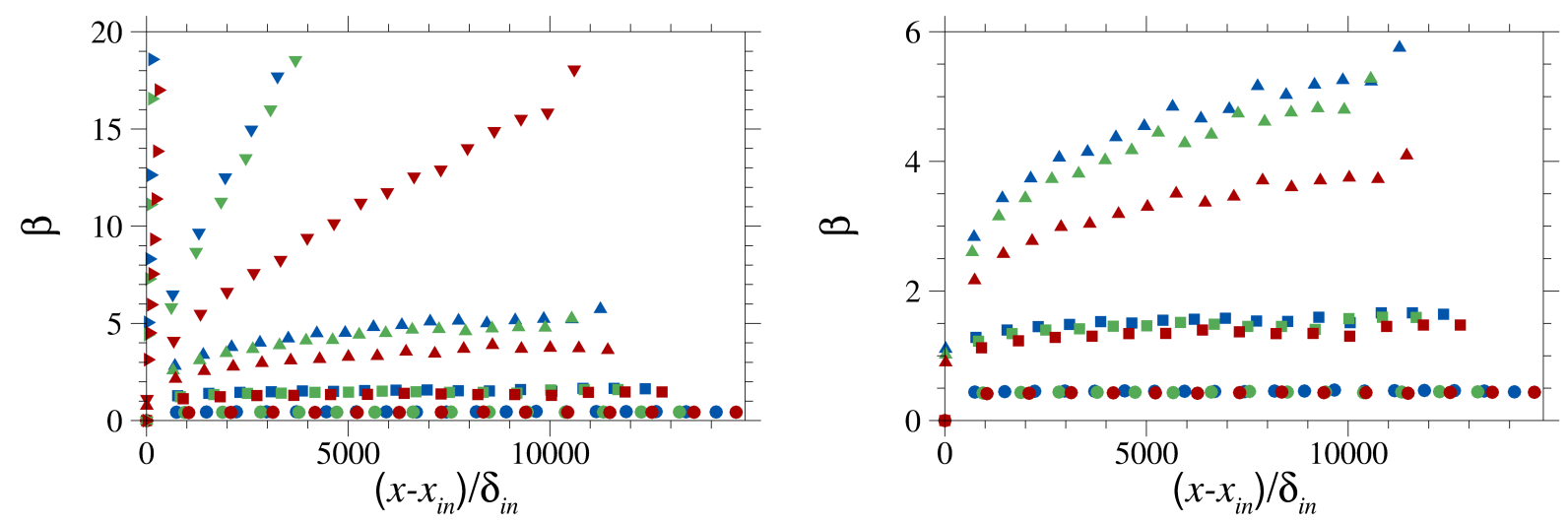

FIG. 4. Streamwise evolution of the Clauser pressure-gradient parameter $\beta$ for the different adversepressure-gradient turbulent boundary layers using different turbulence models: Michel, Quémard, and Durant $^{62}$ (blue), Spalart-Allmaras (green) and $k-\varepsilon$ from Jones and Launder ${ }^{41}$ (red). $\bullet, m=-0.15$; $m=-0.23 ; \mathbf{\Lambda}, m=-0.28 ; \mathbf{\nabla}, m=-0.32 ; \boldsymbol{\vee}, m=-0.4$. Figure (right) corresponds to a zoom of (left).
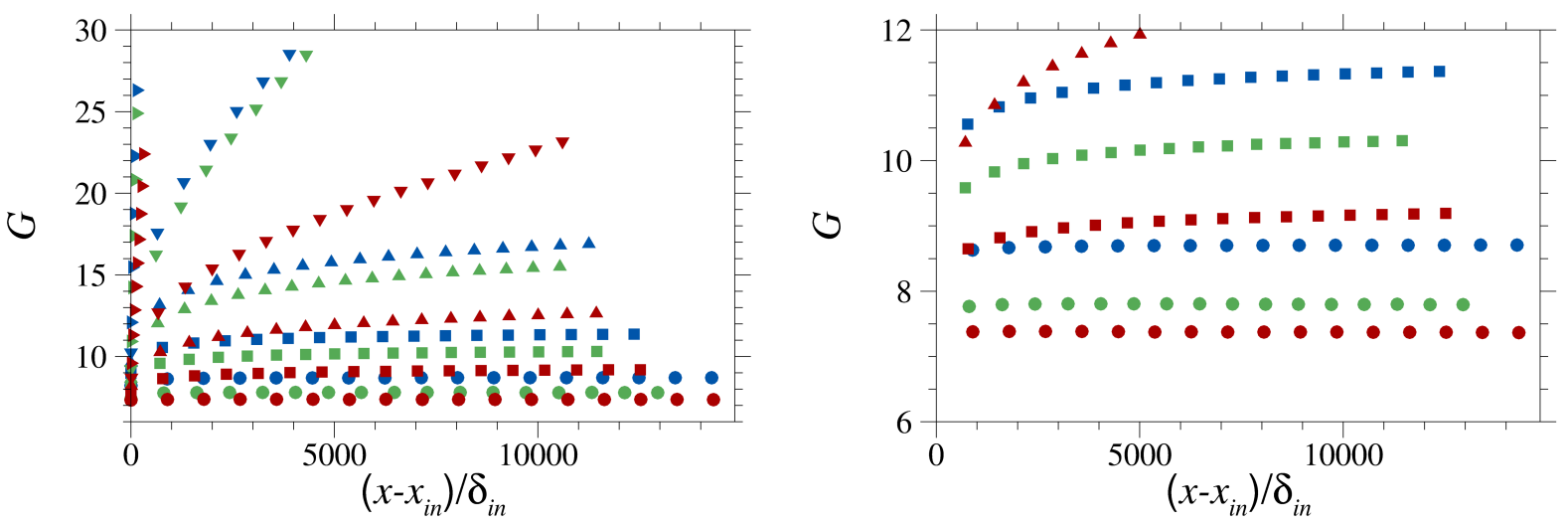

FIG. 5. Streamwise evolution of the Clauser pressure-gradient parameter $G$ for the different adversepressure-gradient turbulent boundary layers using different turbulence models. Symbols and colors as in figure 4. Figure (right) corresponds to a zoom of (left).

small scales and $\tilde{x}_{T} \approx 4$ for parameters dominated by the large scales. For the former, they compare the evolution of the maximum of the Reynolds shear stress in wall units. This maximum is located at $y^{+} \approx 2 \sqrt{\operatorname{Re}_{\tau}}$ for zero-pressure-gradient turbulent boundary layers ${ }^{67-69}$ but at the same time, this position is very close to the location of the very-large-scale motions (VLSM) whose sizes can reach $15 \delta$ in turbulent boundary layers ${ }^{69-72}$. Nevertheless, the Reynolds numbers considered by Sillero, Jiménez, and Moser ${ }^{35}$ are probably not high enough to observe these structures. The origin of integration in their work is taken at $R e_{\theta} \approx 500$ for most of the experiments and simulations studied. 


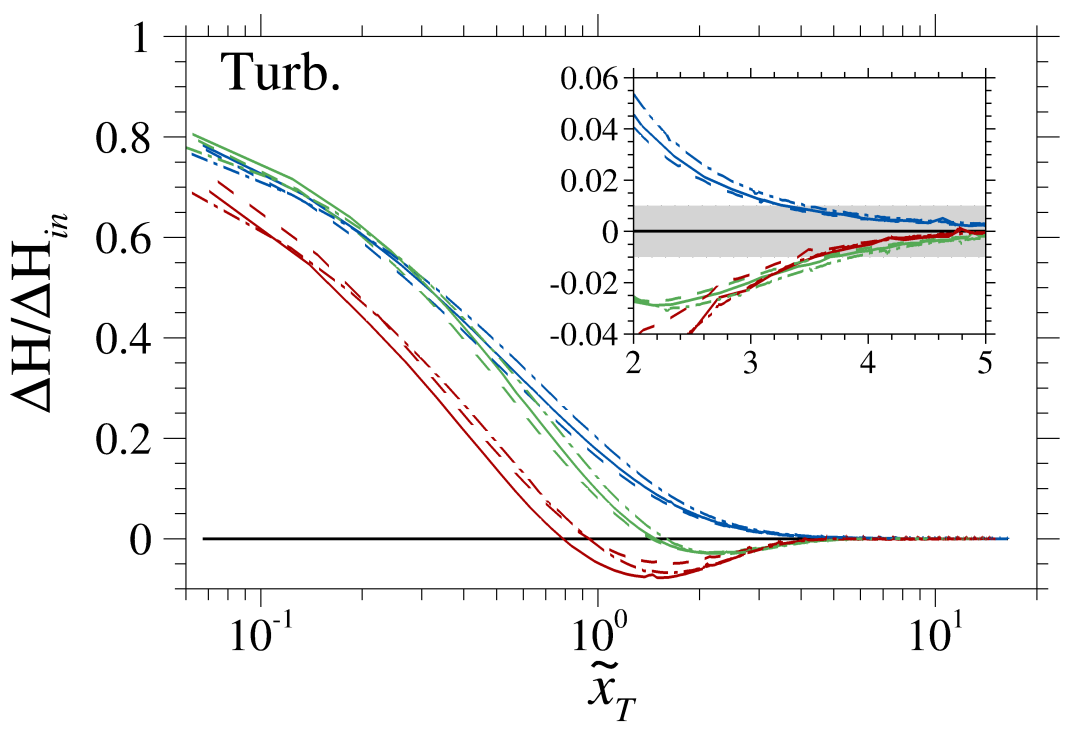

FIG. 6. Streamwise evolution of the shape factor difference $\Delta H / \Delta H_{\text {in }}=\left(H_{p e r}-H_{r e f}\right) /\left(H_{p e r}-H_{r e f}\right)_{i n}$ as a function of $\tilde{x}_{T}$ in a zero-pressure-gradient turbulent boundary layer for different initial shape-factor perturbations and for different turbulence models. Line patterns and colors as in figure 3 (right hand side). The grey area represents $1 \%$ difference. The range of Reynolds number is $\operatorname{Re}_{\theta}$ from $8 \times 10^{3}$ to $10^{6}$.

In addition, $\tilde{x}_{T}$ is also evaluated in a simulation with a higher initial Reynolds number obtaining similar values for convergence thus showing the low Reynolds-number dependence of $\tilde{x}_{T}$ which is an advantage compared to convergence distances based on the boundary layer thickness.

In the present study, the parameter used for the analysis of the perturbation profile effects is the shape factor, which will consequently contain longer history effects than other parameters according to Sillero et al. ${ }^{34}$ and Schlatter and Örlü ${ }^{30}$ as well. Figure 6 shows the evolution of $\Delta H / \Delta H_{\text {in }}$ as a function of $\tilde{x}_{T}$ for the three turbulence models previously mentioned in the case of zero pressure gradient, where it has been chosen $x^{*}=x_{i n}$ for equation (2). As observed, the distances required for convergence to the reference solution in terms of the turnover length are between 3 and 4 (when the shape factor difference reaches $1 \%$ in figure 6), in accordance with the results of Sillero et al. ${ }^{34}$, Sillero, Jiménez, and Moser ${ }^{35}$. Besides, they find that, for the large scales, the corresponding distance in terms of the initial boundary layer thickness is about $200 \delta_{\text {in }}$ for the shape factor, again in accordance with the results in the present study (see figure 3 (right hand side)). 


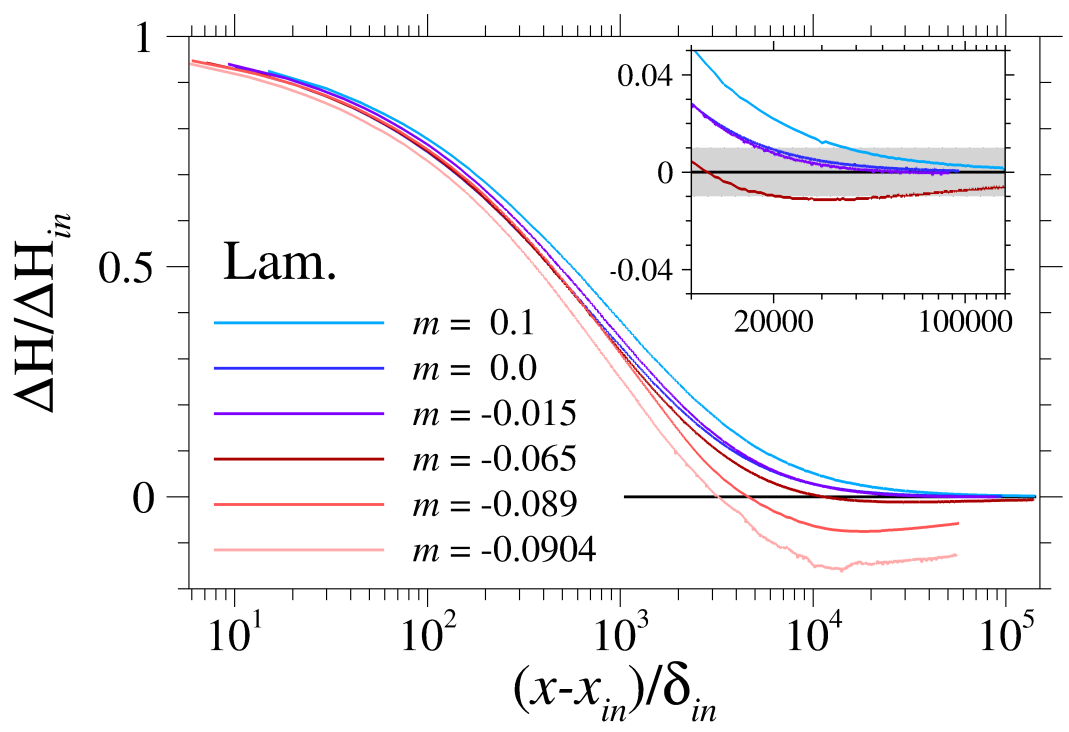

FIG. 7. Streamwise evolution of the shape factor difference in a laminar boundary layer for different external pressure gradients. The grey area represents $1 \%$ difference.

\section{IMPACT OF HISTORY EFFECTS}

\section{A. Convergence length based on the initial boundary layer thickness}

One of the main aspects in analysing the impact of an initial perturbation in the downstream development of the boundary layer is the distance required by the boundary layer to eliminate such effects, if it is possible. This distance can be characterized differently and in the present work it will be done in two different ways. The first one will be based on the initial boundary layer thickness. The use of the boundary layer thickness at a reference station is found in several works $5,7,11,41$. Besides, we believe that, for practical reasons, a distance given in terms of the initial boundary layer thickness may be of interest for researchers, since that parameter is always used to characterize the boundary layer. For instance, in an experimental study, the knowledge of the convergence distance in terms of the boundary layer thickness would definitely influence the experimental set up. Another example could be the turbulent inflow conditions in numerical simulations of turbulent wall-bounded flows in which bigger convergence distances would result in bigger computational domains and hence a greater computational cost.

The evolution of the shape factor difference between the perturbed and the reference boundary layers are presented in figure 7 for laminar boundary layer computations. Only one curve is shown 
for favourable pressure gradient conditions because a lower dependence of $\Delta H / \Delta H_{\text {in }}$ with $m$ has been obtained for $m>0$ than for $m<0$. In all cases shown, the value of the initial perturbation is $\Delta H_{\text {in }} / H_{\text {ref,in }} \approx 1 \%$. One may believe that such a perturbation could be too important but, as presented earlier, results from figure 3 show that the response is essentially independent of the initial perturbation value. For all the pressure gradient intensities studied, the boundary layer seems to converge to the reference case although the convergence distance changes between the different cases. The fastest convergence is observed for the zero-pressure-gradient computation $(m=0)$ and the $m=-0.015$ case reaching the region of $1 \%$ difference at a distance of about $2 \times 10^{4} \delta_{i n}$. The boundary layer profiles are very similar in these two cases with shape factors of 2.59 (corresponding to the value of the Blasius solution ${ }^{10}$ ) and 2.64 respectively. The favorable-pressure-gradient computation $(m=0.1)$ shows a longer convergence distance, of about $4 \times 10^{4} \delta_{i n}$, which is twice the distance for $m=0$. It is interesting to notice that the computations in adverse-pressure-gradient conditions with higher intensities than $m=-0.015$ reach the $1 \%$ difference area faster than the mentioned computations and it happens even faster as the intensity increases. An intersection takes place between the evolution of the perturbed-case shape factor and the reference one when $\Delta H / \Delta H_{\text {in }}=0$ and then the difference in shape factor starts growing again in magnitude but with opposite sign and it leaves the $1 \%$ area before getting into it again much farther downstream for convergence. It could be seen as an overshoot of the perturbed-case shape factor. Besides, as for the $1 \%$ difference area, the intersection point $\left(\Delta H / \Delta H_{\text {in }}=0\right)$ is closer to the origin of the boundary layer as the intensity of the adverse pressure gradient is increased but it does not mean there is a faster convergence as a result of the mentioned overshoot. It seems therefore that the effect of increasing pressure gradient forces is to eliminate faster the shape factor difference. Indeed, a favorable pressure gradient (the case $m=0.1$ ) shows longer convergence distances than the zero pressure gradient case $(m=0)$. However, when a positive pressure gradient is acting on the boundary layer, its effect on the shape factor difference would be too strong to just eliminate the perturbation, as in the favorable and zero pressure gradient computations, leading to the consequent overshoot of $\Delta H / \Delta H_{\text {in }}$ and increase of the convergence length (but reaching faster the $1 \%$ area for the first time). Therefore, the pressure gradient influences the damping of the perturbation making it directly converge to zero for favorable- and zero-pressure-gradients, the shortest distance being obtained for the latter, and to overshoot before convergence in adverse pressure gradient cases. This overshoot increases with the intensity of the pressure gradient thereby enlarging the convergence distance. It is important to point out that in the case $m=-0.015$ an overshoot also 

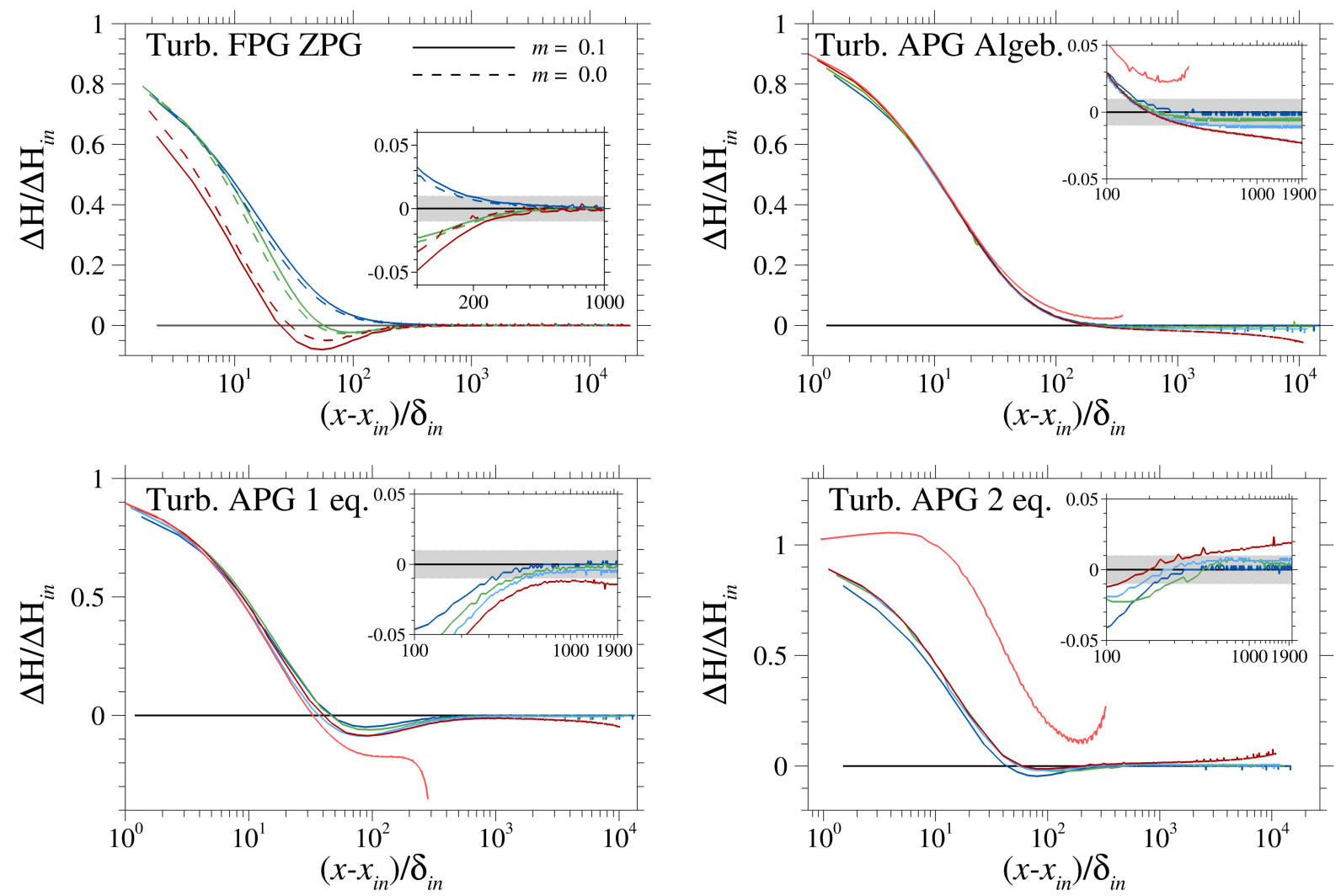

FIG. 8. Streamwise evolution of the shape factor difference in turbulent boundary layers for different external pressure gradients. (Top left) favorable and zero pressure gradients, see figure 3 (right hand side) for line colors and patterns description. Adverse pressure gradient: (Top right), Michel, Quémard, and Durant $^{62}$; (bottom left), Spalart-Allmaras; (bottom right), $k-\varepsilon$ from Jones and Launder ${ }^{41}$. (dark blue), $m=-0.15$; (green), $m=-0.23$; (light blue), $m=-0.28$; (red), $m=-0.32$; (orange), $m=-0.4$. The grey area represents $1 \%$ difference. The range of Reynolds number is $\operatorname{Re}_{\theta}$ from $8 \times 10^{3}$ to $1 \times 10^{6}$ in (top left) and from $8 \times 10^{3}$ to $1.5 \times 10^{6}$ in (top right), (bottom left) and (bottom right).

happens even though it is barely visible in figure 7. This value corresponds to the lowest adversepressure-gradient intensity chosen in the present study for laminar boundary layers. Such a low value might suggest that the overshoot behavior could be observed as soon as adverse pressure gradient forces act on the boundary layer.

The behavior described for laminar boundary layers is less evident in the results for turbulent boundary layers, reported in figure 8 . It is observed that, in the favorable- and zero-pressuregradient cases (figure 8 (top left)), there is again an intersection between the perturbed and reference shape factors evolution when the Spalart-Allmaras and the $k-\varepsilon$ turbulence models are 
considered as well as in the case of the $k-\omega$ SST (not shown). The algebraic model of Michel, Quémard, and Durant ${ }^{62}$ does not show the same behavior than the other models for the case of favorable and zero pressure gradients. This difference may somewhat be explained by the fact that the algebraic model does not rely on transport equations and therefore history effects are included to a lesser extent. When considering adverse pressure gradients (figures 8 (top right, bottom left, bottom right)) all the models used in this study (including the $k-\omega$ SST) show an intersection behavior as well before potential convergence (some cases diverge). Moreover, it is observed that the convergence distance increases as the value of the $m$ exponent decreases (or the intensity of the pressure gradient increases) in adverse pressure gradients, as it is the case for the laminar boundary layer. The behavior in favorable pressure gradients does not seem to follow the same trend for all the models. The convergences distances are quite close to those of $m=0$, with slightly longer distances in favorable pressure gradient for the model of Michel, Quémard, and Durant ${ }^{62}$ and Jones and Launder ${ }^{41}$, which is the behavior observed in laminar boundary layers. In the first model it may again be explained by the fact that history effects are less influencing since turbulent variables do not rely on transport equations.

The intersection behavior observed both in laminar and turbulent boundary layers is more important than it seems. Let us recall that the values of $\Delta H_{\text {in }}$ are small compared to the reference value of the shape factor at $x_{i n}$. The observation of intersections in figures 7 and 8 has led to the definition of the grey area at $1 \%$ as a possible convergence criterion. Such a small criterion might seem excessive compared for example to that given by Chauhan, Monkewitz, and $\mathrm{Nagib}^{36}$ and would be probably not observable in a classical plot of the shape factor evolution, leading to a belief of convergence. However, even within the $1 \%$ area the evolution is in general not converged yet (for those cases in which convergence is achieved) and a total convergence is obtained at larger distances (flat evolution around $\Delta H / \Delta H_{\text {in }}=0$ ). Schlatter and Örlü ${ }^{30}$ analyze small deviations as well in a small part of their work on tripping effects. More particularly, they study the small differences observed in the inner layer of the mean velocity profile for different tripping conditions. They justify studying such small deviations because results from the inner layer in DNS are often used for scaling laws and constants related to this region of the boundary layer because of the difficulty of carrying out accurate measurements in it. Hence, although small deviations might seem to have a lower importance compared to bigger ones, it may have a significant impact on the interpretation of experimental and numerical results.

Getting into a quantitative analysis of the convergence distances obtained, the first thing ob- 
served is the increased damping of the perturbation produced by turbulence. Indeed, the convergence distances for the laminar boundary layer computations are of the order of $2 \times 10^{4} \delta_{\text {in }}$ for $m=0$ and become even larger for both adverse and favorable pressure gradients reaching distances greater than $10^{5} \delta_{\text {in }}$ for boundary layers near separation. The results for turbulent boundary layers show much shorter convergence distances which are of the order of a few hundreds of the inlet boundary layer thickness, similarly to Sillero, Jiménez, and Moser ${ }^{35}$. In their experimental study, Marusic et al. ${ }^{31}$ find convergence distances of the order of $130 \delta_{\text {in }}$ to $200 \delta_{\text {in }}$, but differences in shape factor are still present at those distances of the order of $7 \%$ with respect to the initial difference. In adverse pressure gradient conditions, it is not easy to define very precisely the convergence distance obtained according to the results presented since the behavior of the different models is not the same close to convergence. However all of them show a longer convergence distance for more intense adverse pressure gradients. For the model of Michel, Quémard, and Durant $^{62}$ and that of Jones and Launder ${ }^{41}$, the perturbed-case shape factor crosses the reference one

very close to convergence which is not the case for the model of Spalart and Allmaras ${ }^{64}$. In the latter, the convergence (if achieved) is reached at distances between $300 \delta_{i n}$ and $500 \delta_{i n}$, depending on the value of $m$. Even if it is harder to give convergence distances for the two other models, it can be seen that there are no important differences compared to the results of the Spalart-Allmaras model.

At first glance it could be surprising that laminar boundary layers require larger convergence distances than turbulent boundary layers, keeping in mind that the inner layer of the latter, where viscosity effects are important, converges significantly faster that the outer layer ${ }^{11,30}$. It is wellknown that turbulence increases the mixing and in the case of a turbulent boundary layer it seems intuitive that turbulent diffusion would help to eliminate the differences in the perturbed boundary layer profile and so the convergence of the outer layer would happen faster than convergence in a laminar boundary layer. Concerning the inner layer of the turbulent boundary layer, where viscosity effects are important, the mixing resulting from turbulence in the outer layer leads to larger mean velocity gradients in the inner layer than those observed very near the wall for the laminar boundary layer. As a result, viscosity forces are stronger which could also help to eliminate faster the disturbance in the turbulent boundary layer profile than in that of the laminar boundary layer. 


\section{B. Threshold for convergence}

In the previous section, it has been evoked the difference in the convergence lengths based on the initial boundary layer thickness for both laminar and high-Reynolds-number turbulent boundary layers. It has been shown that the pressure gradient has an impact on these distances. For laminar boundary layers, the fastest convergence is obtained close to $m=0$. As $m$ increases the distance required to eliminate the history effects is increased and the same applies for negative values of $m$ (adverse pressure gradient) when $|m|$ is increased, together with the intersection behavior of the perturbed-case shape factor evolution. Such an increase is observed in the adverse-pressuregradient turbulent boundary layer as well.

However convergence is not obtained for all the computations when $m<0$, at least for the streamwise extent considered. This is somewhat coherent with the fact that the convergence length becomes greater when the intensity of the adverse pressure gradient is larger. There could be a threshold value of the exponent $m_{t h}$ below which convergence is not obtained. It is for that reason that the results are presented for more numerous values of $m$ in adverse pressure gradient conditions for both laminar and turbulent boundary layers.

In the case of laminar boundary layers, the distribution of the external velocity as the streamwise coordinate to the power $m$ corresponds to solutions of the Falkner-Skan equation which are self-similar ${ }^{8,9}$. The most intense adverse pressure gradient that laminar boundary layers in selfsimilarity conditions can resist before boundary layer separation is given by $m \approx-0.0904^{3,9}$. As observed in figure 7 , the impact of the adverse pressure gradient is clearly retarding the convergence but there does not seem to be a pressure gradient leading to non convergence for self-similar laminar boundary layers, and therefore it cannot be defined a value of $m_{t h}$ for these. This is in accordance with the work of Chen and Libby ${ }^{16}$ who show thanks to the linear theory that laminar boundary layers in adverse pressure gradient conditions are spatially stable for $m>m_{\text {sep }}$ (being $m_{\text {sep }}$ the value for boundary layer separation) and for the solutions of positive friction coefficient (let us recall that two solutions exist for the Falkner-Skan equation: one with positive friction coefficient and another one with negative friction coefficient ${ }^{17}$ ).

A different result is clear from figure 8 for turbulent boundary layers. It is first worth noticing the lower values of $m$ (higher absolute value) reached for these boundary layers compared to the laminar boundary layer, showing the well-known capability of the turbulent boundary layer to resist stronger pressure gradients. For all the models shown in figure 8 , the same conclusions 
are observed. As already mentioned, increasing the intensity of the adverse pressure gradient increases the convergence distance, but contrary to the laminar boundary layer, there is a value of the pressure gradient exponent, $m_{t h}$, below which convergence is not possible. This value seems to be between -0.28 and -0.32 for all the models. It is interesting to point out that for all the values of $m$, the perturbed boundary layers start converging towards their respective references, but those where $m<m_{t h}$ end up diverging. Due to the quite long distances required for convergence, this result is important since it shows that in shorter distances it would seem that history effects are almost forgotten whereas it is not strictly the case. Moreover, results for $m=-0.4$ show no convergence and shorter extent because separation happened for all turbulence models.

The threshold value for convergence $m_{t h}$ is very close to the one obtained for the existence of near-equilibrium turbulent boundary layers ${ }^{3,23,24}$. One may conjecture that convergence and equilibrium should be related since near-equilibrium boundary layers give mean velocity and Reynolds stress profiles that are self-similar in the outer layer. Thus, because the external condition given to the boundary layer ( $U_{e}$ distribution) corresponds to a condition of near-equilibrium, the perturbation introduced in the boundary layer will progressively disappear and the parameters influencing the boundary layer profile will be independent of the streamwise location thereby making the boundary layer profile converge to the reference profile. It seems thus that, for the present results, there are not unstable near-equilibrium conditions.

Furthermore, the link between convergence and equilibrium is corroborated for the laminar boundary layer. In accordance with Chen and Libby ${ }^{16}$, it has not been found any value for $m$ for which the boundary layer does not seem to converge and the boundary layer satisfies in all cases the Falkner-Skan equation, i.e. in all cases the boundary layer is in equilibrium. This result is of crucial importance because it shows that turbulent boundary layers in out-of-equilibrium external conditions of the type $U_{e} \propto\left(x-x_{0}\right)^{m}$ (i.e. $m<-0.3$ ) are very dependent on the history effects. It is important to recall that these results are obtained for velocity distributions given by $U_{e} \propto\left(x-x_{0}\right)^{m}$ and so the previous result does not mean that there cannot be any other external velocity distribution different to the present one for which convergence may happen.

\section{Evolution of the perturbed-case shape factor}

All the results presented up to this point are based on small perturbations, in order to get the same behavior of the shape factor difference independently of the initial perturbation. Neverthe- 


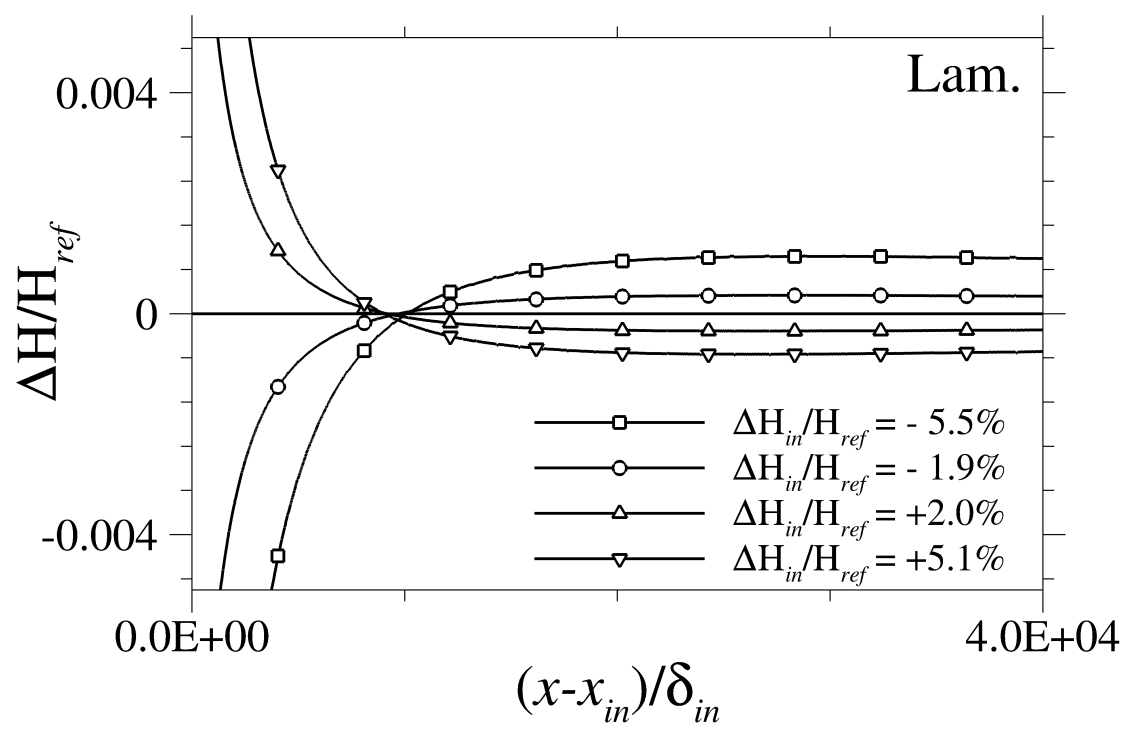

FIG. 9. Streamwise evolution of the shape factor difference in an adverse-pressure-gradient laminar boundary layer with $m=-0.07407$ for different non-small initial shape-factor perturbations.

less, different initial perturbations, not necessarily small, have been tested as well. In particular, figure 9 shows the evolution of the difference between the perturbed-case and the reference-case shape factors non dimensionalized by the local reference shape factor, for $m=-0.07407$ (adverse pressure gradient) in a laminar boundary layer. The different initial values of the perturbed-case shape factor have been chosen to be $\Delta H_{\text {in }} / H_{\text {ref }} \approx \pm 2 \%$ and $\Delta H_{\text {in }} / H_{\text {ref }} \approx \pm 5 \%$. As in the case of small perturbations, boundary layers under adverse pressure gradients show an intersection of the perturbed-case shape factor with the reference one. But what is surprising is the fact that for all the different initial values of the shape factor difference, the evolution of the perturbed-case shape factor crosses that of the reference boundary layer at roughly the same streamwise location, which is about $\left(x-x_{i n}\right) / \delta_{\text {in }} \approx 10^{3}$ for the chosen value of $m$. This behavior is not surprising in the case of small perturbations because the use of these allows to have evolutions of the perturbation that are independent of the initial shape factor difference, as could be demonstrated from the linearized equations. The results reported in figure 9 suggest that laminar boundary layers show an evolution from the perturbed profile independent of the initial perturbation for non-negligible values of this one as well. Consequently, the results obtained in the previous sections regarding the convergence distances in laminar boundary layers also apply for laminar boundary layers experiencing non-negligible perturbations.

In turbulent boundary layers (not shown), this behavior is also in adverse-pressure-gradient 


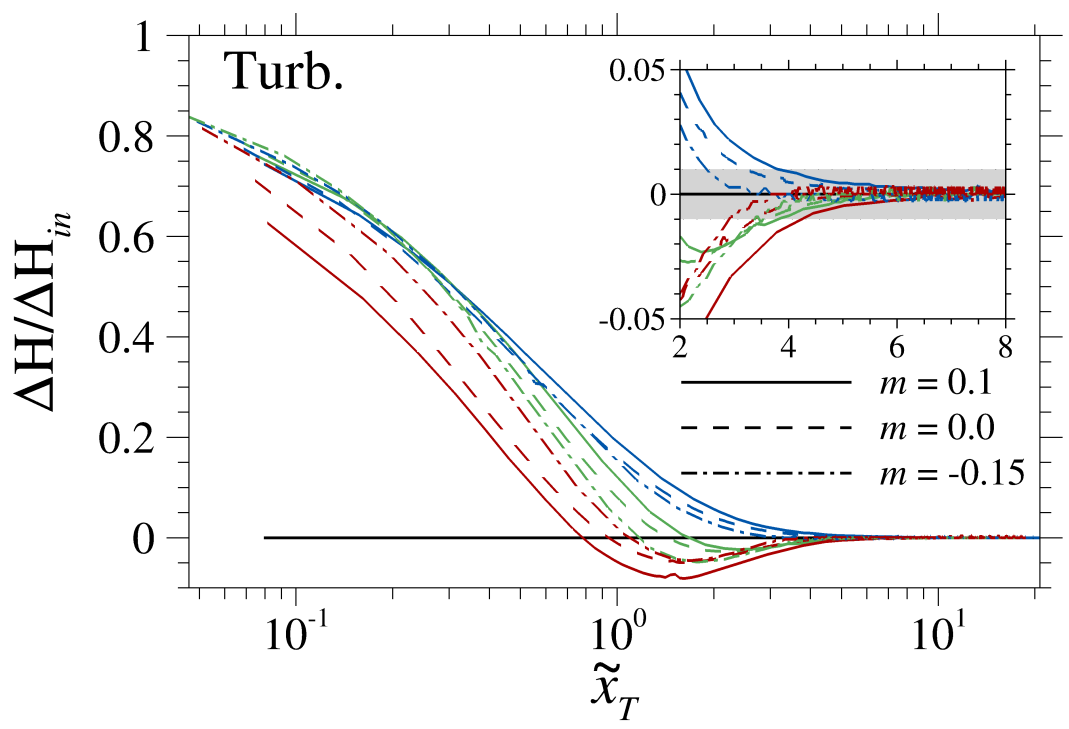

FIG. 10. Streamwise evolution of the shape factor difference as a function of $\tilde{x}_{T}$ for $m=0.1, m=0$ and $m=-0.15$ and different turbulence models (colours as in figure 3). The range of Reynolds number is $R e_{\theta}$ from $8 \times 10^{3}$ to $1.5 \times 10^{6}$.

conditions for the algebraic model of Michel, Quémard, and Durant ${ }^{62}$. The Spalart-Allmaras model shows a similar trend but the streamwise locations of the intersections for different initial perturbations are more widespread. In the case of the Jones-Launder model, the position of the intersection is clearly dependent on the initial value of the perturbation. The locations at which those intersections take place are model-dependent as already observed in figure 8 , with values of about $200 \delta_{\text {in }}$ and $50 \delta_{\text {in }}$ for the algebraic and the one-equation models respectively. This result could be explained by the influence of non-linearities which is more important in one- and twoequations turbulence models. Hence, the conclusions drawn for the laminar boundary layer in the previous paragraph may also be applied to the computations of turbulent boundary layers when using the turbulence model from Michel, Quémard, and Durant ${ }^{62}$ and Spalart and Allmaras ${ }^{64}$. 


\section{CONVERGENCE LENGTH BASED ON BOUNDARY LAYERS CHARACTERISTIC TIMES}

\section{A. Turbulent boundary layers in pressure-gradient conditions}

The use of the turnover time in the present work to characterize the convergence length, as suggested by Simens et al. ${ }^{43}$, has shown similar results for zero-pressure-gradient turbulent boundary layers as other studies in the literature ${ }^{34,35}$. Besides, this criterion has shown to be very appropriate not only because of its physical meaning but also for its low Reynolds-number dependence. In the following, we extend its application to the favorable- and adverse-pressure-gradient turbulent boundary layers.

Turbulent boundary layers under an adverse pressure gradient thicken faster than zero-pressuregradient boundary layers. Moreover, very close to the wall, the mean streamwise momentum equation at leading order (5) shows that the second derivative of the mean streamwise velocity at the wall is positive in adverse pressure gradient boundary layers. Since in attached boundary layers $\partial\langle u\rangle / \partial y>0$ at the wall, the maximum shear takes place at some point away from the wall, contrary to favorable- and zero-pressure-gradient boundary layers ${ }^{58,59}$. The wall-shear stress is therefore lower in adverse pressure gradients as has been observed experimentally and numerically ${ }^{3,54,58,59}$. Thus, the turnover length $L_{T O}=U_{e}^{+} \delta$ tends to be greater for a given streamwise development in turbulent boundary layers subjected to adverse pressure gradients than in those with constant pressure (see equations (2) and (6)). Besides, results from previous sections have shown that the convergence length in terms of the initial boundary layer thickness is greater in adverse pressure gradients for both laminar and turbulent boundary layers and that distance is increased with the intensity of the pressure gradient. Therefore, considering the expression of $\tilde{x}_{T}$ (2), which may be written as

$$
\tilde{x}_{T}=\int_{x^{*}}^{x} \sqrt{\frac{C_{f}}{2}} \frac{\mathrm{d} x}{\delta},
$$

two opposing effects are found in adverse-pressure-gradient conditions with respect to the zeropressure-gradient case. The first one is an increase of $L_{T O}$ due to the lower mean friction coefficient and a faster thickening of in adverse-pressure-gradient turbulent boundary layers which results in a decrease of the integrand in (6) and hence in a decreasing effect in $\tilde{x}_{T}$. The second one is related to the behaviour of the convergence length based on the initial boundary layer thickness $\left(\left[x-x_{i n}\right] / \delta_{i n}\right)$ which becomes larger with the intensity of the adverse pressure gradient. Thus, this second effect 
tends to increase $\tilde{x}_{T}$ because the domain of integration in (6) would be longer. It is a priori not possible to anticipate which of these two effects will have a greater influence.

Figure 10 shows the evolution of the shape factor difference as a function of $\tilde{x}_{T}$. It can be observed that the three turbulence models show values of $\tilde{x}_{T}$ greater and lower than those of zeropressure-gradient computations for the favorable- and adverse-pressure-gradient solutions respectively. For $m=0.1$, this distance is slightly increased with respect to $m=0$, and convergence can be considered for $\tilde{x}_{T} \approx 5$. Considering the adverse-pressure-gradient results, $\tilde{x}_{T}$ decreases when $m$ decreases. Even if, at first, the dependence of $\tilde{x}_{T}$ for convergence with the intensity of the pressure gradient may not make it an appropriate criterion, the values obtained do not change significantly and it can be established that $\tilde{x}_{T}$ should be between 2 and 3 to achieve history effects independence in adverse-pressure-gradient conditions.

\section{B. Laminar boundary layers}

The convergence behavior of laminar boundary layers is now assessed in a similar way than in the previous section through the corresponding characteristic time. In turbulent boundary layers, the viscous diffusion is only important in the viscous sub-layer. Besides, this regions becomes smaller compared to the whole boundary layer thickness as the Reynolds number increases and therefore, for turbulent boundary layers at moderate and high Reynolds numbers, diffusion is dominated by turbulence in most of the boundary layer.

In laminar boundary layers, viscosity is responsible for diffusion and therefore we now must consider the characteristic time based on its effects, which is given by $\delta^{2} / v^{10,73}$. It is possible thereby to define a convergence length for laminar boundary layers equivalent to $\tilde{x}_{T}(2)$ as follows:

$$
\tilde{x}_{L}=\int_{x^{*}}^{x} \frac{\mathrm{d} x}{U_{e} \delta^{2} / v} .
$$

Expression (7) shows the distance of convergence based on the distance the flow travels at a velocity of the order of $U_{e}$ during the characteristic time of viscous effects.

In order to have an estimate of the values that $\tilde{x}_{L}$ should get, let us consider a zero-pressuregradient laminar boundary layer, for which the solution of the Blasius' equations gives ${ }^{10}$ :

$$
\frac{\delta}{x} \approx 4.92 R e_{x}^{-\frac{1}{2}}
$$

If we consider a boundary layer developing over a flat plate along a streamwise distance $L$ with an 
external velocity $U_{\infty}$, it is possible to write:

$$
\frac{1}{\delta(L)^{2} U_{\infty} / v} \int_{0}^{L} \mathrm{~d} x=\frac{1}{4.92^{2}} \approx 0.041
$$

which is independent of $L$ and therefore for a zero-pressure-gradient laminar boundary layer it must be $\tilde{x}_{L} \sim 10^{-2}$, because in equation (7) $\delta$ is the local boundary layer thickness and it increases with $x$. Besides, from the Falkner-Skan solution for $U_{e} \propto x^{m}$ :

$$
\frac{1}{\delta^{2} U_{e} / v}=\frac{m+1}{2 \eta_{99}^{2}(m) x}=\frac{m+1}{2 \eta_{99}^{2}(m) x / \delta_{i n}} \frac{1}{\delta_{i n}}
$$

where $\eta_{99}$ is the wall normal coordinate of the Falkner-Skan solution for which $u=0.99 U_{e}$ and it decreases with the exponent $m^{9,10}$. As shown in figure 7, the distance for convergence in terms of $\delta_{\text {in }}$ increases for adverse-pressure-gradient boundary layers when the intensity of the pressure gradient is increased. Since $\eta_{99}(m)$ decreases with $m$ for $m<0$ (adverse pressure gradient) and it is positive, $\eta_{99}^{2}(m)$ also decreases with $m$. In addition, all the values of $\delta_{i n}$ in the present computations have been chosen to be of the same order of magnitude therefore the term $\left(\delta^{2} U_{e} / v\right)^{-1}$ in equation (10) should be lower for smaller values of $m$, i.e. for more intense adverse pressure gradients. In the case of favourable pressure gradients, it cannot be anticipated the evolution of $\tilde{x}_{L}$ with the intensity of the pressure gradient due to the increase of the convergence length based on $\delta_{i n}$ when the favourable pressure gradient is stronger.

Results of the laminar boundary layer computations using $\tilde{x}_{L}$ are reported in figure 11 for favorable-, adverse- and zero-pressure-gradient laminar boundary layers. Just as in the turbulent boundary layer simulations, it has been chosen $x^{*}=x_{i n}$. For $m=0$, convergence is reached when $\tilde{x}_{L}$ is about 0.08 which is of the order of $10^{-2}$ as expected. Regarding the solutions for $m<0$, the distance for convergence decreases for $m=-0.015$ according to the previous reasoning but it is not the case for $m=-0.065$. This is due to the larger convergence distance $\left(x-x_{i n}\right) / \delta_{\text {in }}$ required for this computation (figure 7$)$. In fact, $\left(x-x_{i n}\right) / \delta_{\text {in }}$ decreases the integrand as shown in equation (10) but at the same time it increases the integration distance in (7). Nevertheless, the beginning of the curve follows the predictions of the previous reasoning (the curve tends to zero faster). In the case of the favorable pressure gradient, $\tilde{x}_{L}$ is also larger than for $m=0$ with convergence distances $\tilde{x}_{L} \approx 0.1$ which should increase if $m$ is increased, since it is the trend observed for convergence distances based on the initial boundary layer thickness.

Therefore, the convergence length based on the characteristic time of the laminar boundary layer shows values that are dependent on the pressure gradient, just like $\tilde{x}_{T}$ in turbulent boundary 


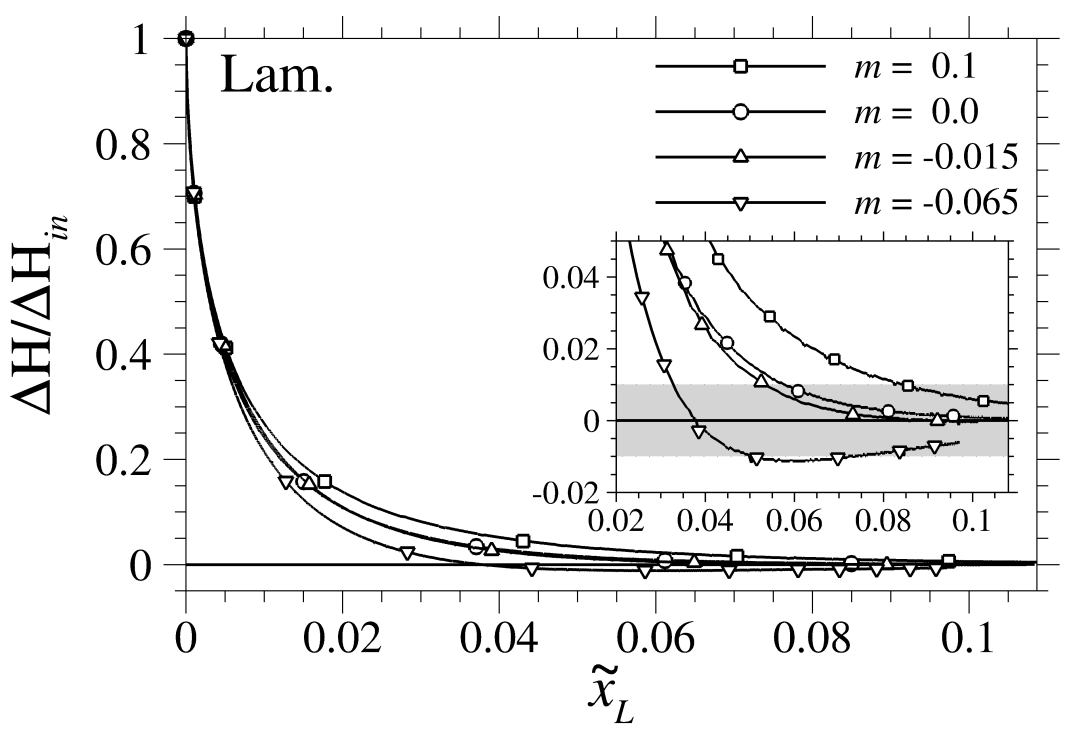

FIG. 11. Streamwise evolution of the shape factor difference in laminar boundary layers in favourable, adverse and zero pressure gradients. The grey area represents $1 \%$ difference.

layers. Despite such dependency, a value can be given for zero-pressure-gradient laminar boundary layers $\left(\tilde{x}_{L} \sim 0.08\right)$ and for favorable and adverse pressure gradients the values obtained are not far from that of zero pressure gradient. This parameter can consequently be used to evaluate whether a laminar boundary layer is free from history effects in a similar way $\tilde{x}_{T}$ does for turbulent boundary layers.

\section{CONCLUSIONS}

The aim of the present work has been to contribute to the understanding of the history effects in boundary layers by means of perturbations of the upstream boundary condition. History effects are present in most of the engineering applications as well as in experimental studies and numerical simulations of boundary layers. Many research works give few or no information about the upstream development of the boundary layers before the region of interest and it has been shown that history effects may lead to important differences in the analysis of the results ${ }^{30}$.

The shape factor has been the main parameter used for the present study. Different boundary layers have been analyzed with different pressure gradient evolutions, all of them seeking to reproduce equilibrium conditions in both laminar and turbulent conditions. The boundary layers have been simulated from an inlet boundary layer profile which has been perturbed and it is the 
evolution of this perturbation that has been evaluated by means of the shape factor.

The present study has given a more quantitative idea about the convergence distances (i.e. the distance required to eliminate history effects) based on the initial boundary layer thickness which is a quantity easily obtained. Convergence distances in the literature are mainly studied in zeropressure-gradient conditions whereas in the present work they are compared between favorable-, zero- and adverse-pressure-gradient conditions for both laminar and fully turbulent boundary layers. Besides, a second convergence distance is used, based on the main physical phenomenon in play respectively in laminar and turbulent boundary layers. In the case of turbulent boundary layers, this distance already existed in the literature (and it has been used for zero-pressure-gradient conditions) whereas it has been proposed in laminar boundary layers for the first time to the authors' best knowledge.

The effects of the history of the boundary layer (the presence of the perturbation introduced) have shown to be important in a quite large streamwise extent. In terms of the initial boundary layer thickness, it has been observed that laminar boundary layers do not eliminate these effects until very long distances, of the order of at least $2 \times 10^{4} \delta_{i n}$. This distance is dependent on the exponent $m$ of the external velocity distribution, being minimum for $m \approx 0$ and increasing for any other value. Besides, it has also been observed that the pressure gradient for laminar boundary layers creates an overshoot in the evolution of the shape factor difference for the adverse-pressuregradient conditions which is more pronounced as the intensity of the pressure gradient is raised.

In turbulent boundary layers, the convergence distance based on the initial boundary layer thickness is significantly shorter, although it still represents quite long distances. For zero-pressuregradient conditions, distances of about $200 \delta_{\text {in }}$ or $300 \delta_{\text {in }}$ are required for convergence of the shape factor difference. This distance evolves with the pressure gradient, increasing for adverse pressure gradients to distances that can reach up to $500 \delta_{i n}$. In the case of favorable pressure gradients, the evolution of the convergence distance with the pressure gradient intensity is not very clear because the different turbulence models do not behave exactly the same way. However it seems that the distances do not change significantly and are of the same order as in zero-pressure-gradient conditions.

The intensity of the pressure gradient beyond which convergence is not possible has also been studied. In fact, in adverse-pressure-gradient conditions the convergence distance increases as the intensity increases (or as $m$ decreases) for both the laminar and the turbulent computations. The first one does not show any threshold for $m$, whereas the second one does not show convergence 
when $m<-0.3$. This value is the same threshold for obtaining near-equilibrium conditions. The fact that laminar boundary layers seem to converge for all the adverse pressure gradient computations supports the idea that equilibrium and convergence are related aspects.

The convergence distance has also been addressed from a different definition given by Sillero et al. ${ }^{34}$, which, for turbulent boundary layers, relates the distance to the turnover time $\delta / u_{\tau}$. It has been obtained from the present results that this distance, here denoted $\tilde{x}_{T}$, is of the order of 5 in the favorable pressure gradient computations, whereas it decreases to between 2 and 3 for the adverse pressure gradient simulations. In zero-pressure-gradient conditions the value obtained is between 3 and 4. Although the values for convergence are dependent on the pressure gradient, they remain of the same order of magnitude which makes $\tilde{x}_{T}$ a quite good indicator for convergence from upstream effects.

An equivalent convergence distance to $\tilde{x}_{T}$, denoted $\tilde{x}_{L}$ has been proposed for laminar boundary layers based on its characteristic time which is given by viscous diffusion $\left(\delta^{2} / v\right)$. This criterion has shown to be of the order of 0.08 for zero-pressure-gradient boundary layers. Again, the pressure gradient modifies the values of this convergence distance: in the case of favorable pressure gradients, values of $\tilde{x}_{L} \sim 0.1$ are observed, and also similar values to zero-pressure-gradient conditions are obtained for adverse-pressure-gradient laminar boundary layers.

It is important to bear in mind that the conclusions drawn in the present study are based on the case of small perturbations and for external velocity distributions given by a power law. Additional studies considering more generic conditions would be of huge interest. Besides, the present one focuses only on the boundary layer equations and more results of higher fidelity approaches such as DNS or experiments would be essential to complement the present conclusions. Nevertheless, such approaches remain for the moment more limited in Reynolds number because of the cost of the computation or of the experimental setup.

An interesting point concluded in the present work is that the convergence from history effects seems to be quite universal in terms of the pressure gradient. Except for the case of very strong adverse pressure gradients in turbulent boundary layers, convergence has always been obtained in the present study. A pressure-gradient dependent behavior of convergence would lead to important issues in turbulence modeling. For instance, if a non-convergence behavior i.e. sensitivity to upstream conditions was obtained under adverse pressure gradients, then it would not be possible to observe a canonical flow because of its instability, which would make the task of universal wall-bounded turbulence modeling almost intractable. Despite these favorable results of conver- 
gence behavior, it has been observed that very large distances to achieve convergence are required based on the criteria presented making the observation of canonical flows not trivial and giving importance to the modeling of relaxation towards these flows.

\section{ACKNOWLEDGMENTS}

The authors are greatly thankful to all the people involved in the evolution of the CLICET code. The thesis of J. Vaquero is partly funded by DGA (French defense procurement agency). Support from the framework of the ONERA research project FROTTEMENT is also acknowledged.

\section{Appendix A: Description of the numerical tool and validation in pressure gradient conditions}

In the following, the equations employed in the solver CLICET are presented, as described by Aupoix $^{48} . x$ and $y$ are the streamwise and wall-normal directions respectively and $u$ and $v$ their corresponding velocity components. Body forces are not considered in the present study. The Reynolds average of a given quantity $f$ is written $\langle f\rangle$, and so it is possible to decompose $f$ as $f=\langle f\rangle+f^{\prime}$ where $f^{\prime}$ is the fluctuating part. The mass-weighted mean value of a given quantity is considered to take into account compressibility effects (negligible in the present study). One may write:

$$
\tilde{f}=\frac{\langle\rho f\rangle}{\langle\rho\rangle}, \quad f=\tilde{f}+f^{\prime \prime}
$$

Thus, the boundary layer equations are:

$$
\begin{gathered}
\frac{\partial}{\partial x}(\langle\rho\rangle \tilde{u})+\frac{\partial}{\partial y}(\langle\rho\rangle \tilde{v})=0 \\
\langle\rho\rangle \tilde{u} \frac{\partial \tilde{u}}{\partial x}+\langle\rho\rangle \tilde{v} \frac{\partial \tilde{u}}{\partial y}=-\frac{\mathrm{d}\langle p\rangle}{\mathrm{d} x}+\frac{\partial}{\partial y}\left(\mu \frac{\partial \tilde{u}}{\partial y}-\left\langle\rho u^{\prime \prime} v^{\prime \prime}\right\rangle\right) \\
0=-\frac{\partial\langle p\rangle}{\partial y} \\
\langle\rho\rangle \tilde{u} \frac{\partial \tilde{h}_{i}}{\partial x}+\langle\rho\rangle \tilde{v} \frac{\partial \tilde{h}_{i}}{\partial y}=\frac{\partial}{\partial y}\left(\tilde{u}\left(\mu \frac{\partial \tilde{u}}{\partial y}-\left\langle\rho u^{\prime \prime} v^{\prime \prime}\right\rangle\right)\right)+ \\
\frac{\partial}{\partial y}\left(\lambda \frac{\partial \tilde{T}}{\partial y}-\left\langle\rho v^{\prime \prime} h^{\prime \prime}\right\rangle\right) \\
\langle p\rangle=\langle\rho\rangle R_{g} \tilde{T}
\end{gathered}
$$


where $h_{i}$ and $h$ are the stagnation and static enthalpies respectively which, under the boundary layer hypothesis, are related to the mean streamwise velocity: $\tilde{h}_{i}=\tilde{h}+\tilde{u}^{2} / 2$. Regarding boundary conditions, the no-slip adiabatic wall together with the link to the external flow conditions give:

$$
\begin{array}{lllll}
u=0, v=0 & \text { and } & \frac{\partial T}{\partial y}=0 & \text { on } & y=0 \\
u \rightarrow U_{e}(x) & \text { and } & T \rightarrow T_{e} & \text { as } & y \rightarrow \infty .
\end{array}
$$

The pressure is known from the external pressure evolution $p_{e}(x)$ according to equation (A4). Thus, the density is obtained from the equation of state (A6) and, since $h=c_{p} T$ ( $c_{p}$ being the specific heat at constant pressure), only boundary conditions on velocity and temperature are required.

Although the solver CLICET has already been used for other studies ${ }^{49,50}$, an additional validation test has been performed specifically for this study for boundary layers experiencing adversepressure-gradient conditions. The experiment considered is that given in the work of Harun ${ }^{74}$. In this one, a boundary layer under an adverse pressure gradient of almost constant pressure coefficient gradient $\left(\mathrm{d} C_{p} / \mathrm{d} x\right)$ is studied. The external velocity according to the given pressure coefficient evolution is given in figure 12. The boundary layer is in turbulent conditions, therefore different models are considered for the computations: the algebraic model of Michel, Quémard, and Durant $^{62}$, the one equation model of Spalart and Allmaras ${ }^{64}$ and the $k-\varepsilon$ two-equation model of Jones and Launder ${ }^{41}$. The results for the shape factor are presented in figure 13. A fairly good agreement between numerical results and experimental data is observed confirming the capability of the solver to reproduce boundary layers in adverse-pressure-gradient conditions. Besides, comparisons have been made between the solutions of the boundary layer equations and the full Navier-Stokes equations in the case of a boundary layer under an adverse pressure gradient leading to separation. These comparisons have been made until right before separation showing again a satisfactory agreement between both solutions.

\section{REFERENCES}

${ }^{1}$ I. Tani, “Boundary-layer transition,” Annual Review of Fluid Mechanics 1, 169-196 (1969). 
Upstream perturbations effects on laminar and turbulent boundary layers

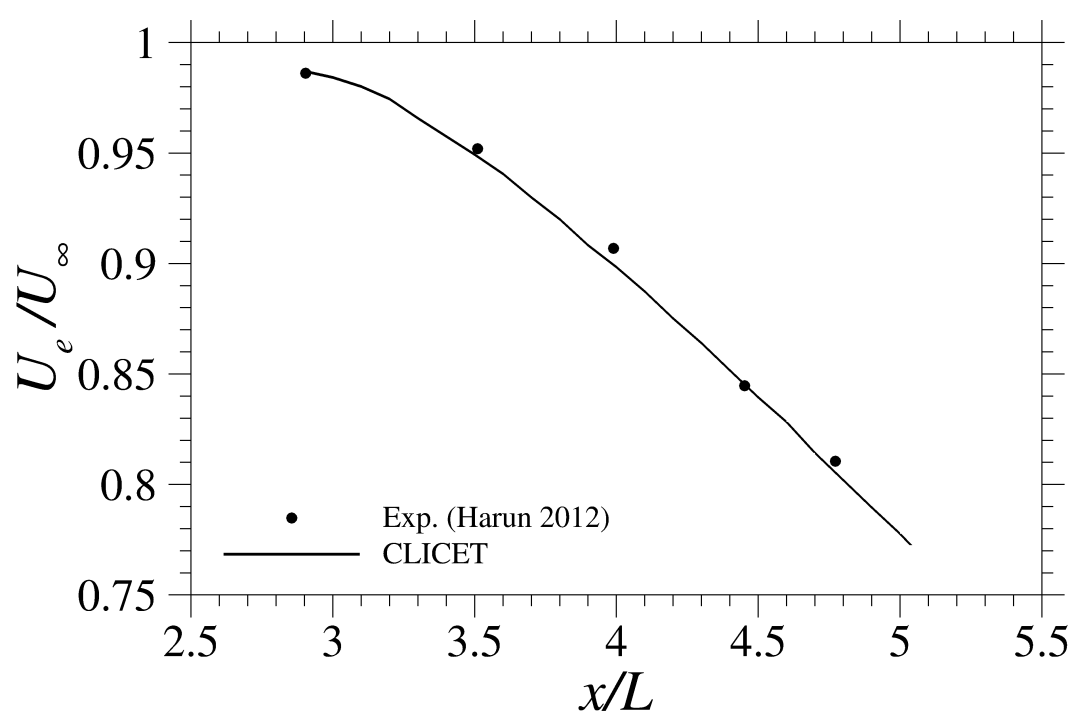

FIG. 12. External velocity evolution. Experimental data from Harun ${ }^{74}$.

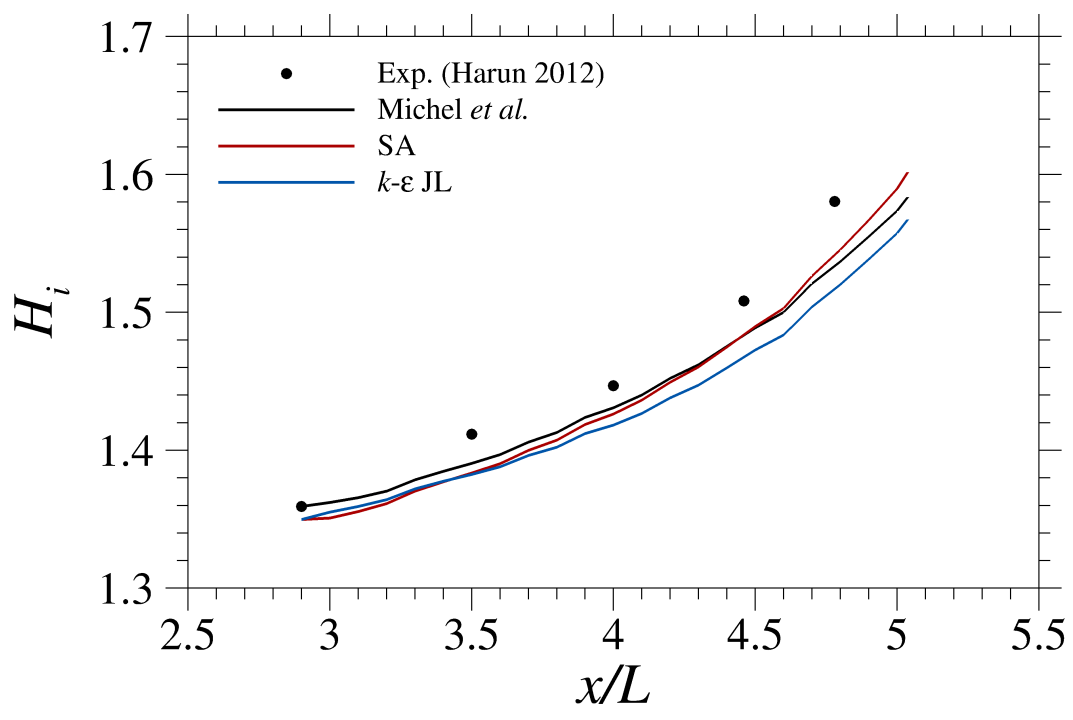

FIG. 13. Shape factor evolution for different turbulence models from Michel, Quémard, and Durant ${ }^{62}$, Spalart and Allmaras ${ }^{64}$ and Jones and Launder ${ }^{41}$. Experimental data from Harun ${ }^{74}$.

${ }^{2}$ P. Sagaut, S. Deck, and M. Terracol, Multiscale and Multiresolution Approaches in Turbulence, 2nd ed. (Imperial College Press, 2013).

${ }^{3}$ F. H. Clauser, "Turbulent boundary layers in adverse pressure gradients," Journal of the Aeronautical Sciences 21, 91-108 (1954).

${ }^{4}$ R. Vinuesa, P. H. Rozier, P. Schlatter, and H. M. Nagib, "Experiments and computations of 
localized pressure gradients with different history effects," AIAA Journal 52, 368-384 (2014).

${ }^{5}$ A. Bobke, R. Vinuesa, R. Örlü, and P. Schlatter, "History effects and near equilibrium in adverse-pressure-gradient turbulent boundary layers," Journal of Fluid Mechanics 820, 667-692 (2017).

${ }^{6}$ R. Vinuesa, R. Örlü, C. Sanmiguel Vila, A. Ianiro, S. Discetti, and P. Schlatter, "Revisiting history effects in adverse-pressure-gradient turbulent boundary layers," Flow, Turbulence and Combustion 99, 565-587 (2017).

${ }^{7}$ A. J. Smits and D. H. Wood, "The response of turbulent boundary layers to sudden perturbations," Annual Review of Fluid Mechanics 17, 321-358 (1985).

${ }^{8}$ V. Falkner and M. S. Skan, "LXXXV. Solutions of the boundary-layer equations," The London, Edinburgh, and Dublin Philosophical Magazine and Journal of Science 12, 865-896 (1931).

${ }^{9}$ D. R. Hartree, “On an equation occurring in Falkner and Skan's approximate treatment of the equations of the boundary layer," Mathematical Proceedings of the Cambridge Philosophical Society 33, 223 (1937).

${ }^{10}$ H. Schlichting, Boundary-Layer Theory, edited by McGraw-Hill (1968).

${ }^{11}$ F. H. Clauser, "The turbulent boundary layer," in Advances in Applied Mechanics (Elsevier, 1956) pp. 1-51.

${ }^{12}$ W. F. Gallo, A. V. Gnos, and J. G. Marvin, "Nonsimilar nature of the laminar boundary layer," AIAA Journal 8, 75-81 (1970).

${ }^{13}$ J. G. Marvin and A. R. Sinclair, "Convective heating in regions of large favorable pressure gradient.” AIAA Journal 5, 1940-1948 (1967).

${ }^{14}$ J. Serrin, "Asymptotic behaviour of velocity profiles in the prandtl boundary layer theory," Proceedings of the Royal Society A: Mathematical, Physical and Engineering Sciences 299, 491507 (1967).

${ }^{15}$ L. A. Peletier, "On the asymptotic behaviour of velocity profiles in laminar boundary layers," Archive for Rational Mechanics and Analysis 45, 110-119 (1972).

${ }^{16}$ K. K. Chen and P. A. Libby, "Boundary layers with small departures from the Falkner-Skan profile," Journal of Fluid Mechanics 33, 273 (1968).

${ }^{17}$ P. A. Libby and T. M. Liu, "Further solutions of the Falkner-Skan equation." AIAA Journal 5, 1040-1042 (1967).

${ }^{18}$ J. Rotta, “Turbulent boundary layers in incompressible flow," Progress in Aerospace Sciences 2, $1-95$ (1962). 
${ }^{19}$ G. L. Mellor and D. M. Gibson, "Equilibrium turbulent boundary layers,” Journal of Fluid Mechanics 24, 225 (1966).

${ }^{20} \mathrm{~A}$. A. Townsend, The structure of turbulent shear flow (Cambridge University Press, Cambridge England New York, 1976).

${ }^{21}$ C. Bourassa and F. Thomas, "An experimental investigation of a highly accelerated turbulent boundary layer," Journal of Fluid Mechanics 634, 359 (2009).

${ }^{22}$ S. Dixit and O. Ramesh, "Large-scale structures in turbulent and reverse-transitional sink flow boundary layers," Journal of Fluid Mechanics 649, 233-273 (2010).

${ }^{23}$ M. R. Head, "Equilibrium and near-equilibrium turbulent boundary layers," Journal of Fluid Mechanics 73, 1 (1976).

${ }^{24}$ W. H. Schofield, "Equilibrium boundary layers in moderate to strong adverse pressure gradients," Journal of Fluid Mechanics 113, 91 (1981).

${ }^{25}$ A. E. Perry and W. H. Schofield, "Mean velocity and shear stress distributions in turbulent boundary layers," Physics of Fluids 16, 2068 (1973).

${ }^{26}$ J. H. Lee, "Large-scale motions in turbulent boundary layers subjected to adverse pressure gradients," Journal of Fluid Mechanics 810, 323-361 (2017).

${ }^{27}$ B. S. Stratford, “An experimental flow with zero skin friction throughout its region of pressure rise," Journal of Fluid Mechanics 5, 17 (1959).

${ }^{28}$ V. Kitsios, A. Sekimoto, C. Atkinson, J. A. Sillero, G. Borrell, A. G. Gungor, J. Jiménez, and J. Soria, "Direct numerical simulation of a self-similar adverse pressure gradient turbulent boundary layer at the verge of separation," Journal of Fluid Mechanics 829, 392-419 (2017).

${ }^{29}$ L. P. Erm and P. N. Joubert, "Low-Reynolds-number turbulent boundary layers," Journal of Fluid Mechanics 230, 1 (1991).

${ }^{30} \mathrm{P}$. Schlatter and R. Örlü, "Turbulent boundary layers at moderate Reynolds numbers: inflow length and tripping effects," Journal of Fluid Mechanics 710, 5-34 (2012).

${ }^{31}$ I. Marusic, K. A. Chauhan, V. Kulandaivelu, and N. Hutchins, "Evolution of zero-pressuregradient boundary layers from different tripping conditions," Journal of Fluid Mechanics 783, 379-411 (2015).

${ }^{32} \mathrm{P}$. Schlatter and R. Örlü, “Assessment of direct numerical simulation data of turbulent boundary layers,” Journal of Fluid Mechanics 659, 116-126 (2010).

${ }^{33}$ C. Sanmiguel Vila, R. Vinuesa, S. Discetti, A. Ianiro, P. Schlatter, and R. Örlü, "On the identification of well-behaved turbulent boundary layers," Journal of Fluid Mechanics 822, 109-138 
Upstream perturbations effects on laminar and turbulent boundary layers

(2017).

${ }^{34}$ J. Sillero, J. Jiménez, R. Moser, and N. P. Malaya, "Direct simulation of a zero-pressure-gradient turbulent boundary layer up to $R e_{\theta}=6650$," Journal of Physics: Conference Series 318, 022023 (2011).

${ }^{35}$ J. A. Sillero, J. Jiménez, and R. D. Moser, "One-point statistics for turbulent wall-bounded flows at Reynolds numbers up to $\delta^{+} \approx 2000$," Physics of Fluids 25, 105102 (2013).

${ }^{36}$ K. A. Chauhan, P. A. Monkewitz, and H. M. Nagib, "Criteria for assessing experiments in zero pressure gradient boundary layers," Fluid Dynamics Research 41, 021404 (2009).

${ }^{37}$ P. H. Alfredsson, A. Segalini, and R. Örlü, "A new scaling for the streamwise turbulence intensity in wall-bounded turbulent flows and what it tells us about the 'outer' peak," Physics of Fluids 23, 041702 (2011).

${ }^{38}$ L. Castillo and T. G. Johansson, "The effects of the upstream conditions on a low Reynolds number turbulent boundary layer with zero pressure gradient," Journal of Turbulence 3, N31 (2002).

${ }^{39}$ J. Seo, L. Castillo, T. G. Johansson, and H. Hangan, "Reynolds stress in turbulent boundary layers at high Reynolds number," Journal of Turbulence 5 (2004), 10.1088/1468-5248/5/1/015.

${ }^{40}$ H. J. Herring and J. F. Norbury, "Some experiments on equilibrium turbulent boundary layers in favourable pressure gradients," Journal of Fluid Mechanics 27, 541 (1967).

${ }^{41}$ W. P. Jones and B. E. Launder, "Some properties of sink-flow turbulent boundary layers,” Journal of Fluid Mechanics 56, 337 (1972).

${ }^{42}$ P. N. Inman and P. Bradshaw, "Mixing length in low Reynolds number turbulent boundary layers," AIAA Journal 19, 653-655 (1981).

${ }^{43}$ M. P. Simens, J. Jiménez, S. Hoyas, and Y. Mizuno, “A high-resolution code for turbulent boundary layers," Journal of Computational Physics 228, 4218-4231 (2009).

${ }^{44}$ A. E. Perry, I. Marušić, and J. D. Li, "Wall turbulence closure based on classical similarity laws and the attached eddy hypothesis," Physics of Fluids 6, 1024-1035 (1994).

${ }^{45}$ A. E. Perry and I. Marusic, "A wall-wake model for the turbulence structure of boundary layers. Part 1. Extension of the attached eddy hypothesis," Journal of Fluid Mechanics 298, 361 (1995).

${ }^{46}$ I. Marusic and A. E. Perry, "A wall-wake model for the turbulence structure of boundary layers. Part 2. Further experimental support," Journal of Fluid Mechanics 298, 389 (1995).

${ }^{47}$ A. E. Perry, I. Marusic, and M. B. Jones, "On the streamwise evolution of turbulent boundary layers in arbitrary pressure gradients," Journal of Fluid Mechanics 461 (2002), 


\section{$10.1017 / \mathrm{s} 002211200200825 x$.}

${ }^{48}$ B. Aupoix, "Couches Limites Bidimensionnelles Compressibles. Descriptif et Mode d'emploi du code CLICET - Version 2005," Tech. Rep. (RT 17/05284, ONERA, 2005).

${ }^{49}$ B. Aupoix and P. Spalart, "Extensions of the Spalart-Allmaras turbulence model to account for wall roughness," International Journal of Heat and Fluid Flow 24, 454-462 (2003).

${ }^{50}$ N. Renard and S. Deck, "A theoretical decomposition of mean skin friction generation into physical phenomena across the boundary layer," Journal of Fluid Mechanics 790, 339-367 (2016).

${ }^{51}$ S. Deck, N. Renard, R. Laraufie, and P.-É. Weiss, "Large-scale contribution to mean wall shear stress in high-Reynolds-number flat-plate boundary layers up to $R e_{\theta}=13650$," Journal of Fluid Mechanics 743, 202-248 (2014).

${ }^{52}$ M. Inoue, R. Mathis, I. Marusic, and D. I. Pullin, "Inner-layer intensities for the flat-plate turbulent boundary layer combining a predictive wall-model with large-eddy simulations," Physics of Fluids 24, 075102 (2012).

${ }^{53}$ S. Deck, N. Renard, R. Laraufie, and P. Sagaut, "Zonal Detached Eddy Simulation (ZDES) of a spatially developing flat plate turbulent boundary layer over the Reynolds number range 3150 $\leq R e_{\theta} \leq 14000$," Physics of Fluids 26, 025116 (2014).

${ }^{54}$ P. E. Skåre and P.-A. Krogstad, “A turbulent equilibrium boundary layer near separation,” Journal of Fluid Mechanics 272, 319 (1994).

${ }^{55}$ M. Skote, D. Henningson, and R. Henkes, "Direct numerical simulation of self-similar turbulent boundary layers in adverse pressure gradient conditions," Flow, Turbulence and Combustion 60, 47-85 (1998).

${ }^{56}$ J. Monty, Z. Harun, and I. Marusic, “A parametric study of adverse pressure gradient turbulent boundary layers," International Journal of Heat and Fluid Flow 32, 575-585 (2011).

${ }^{57}$ H. Nagib and K. Chauhan, "Variations of von Kármán coefficient in canonical flows," Physics of Fluids 20, 101518 (2008).

${ }^{58} \mathrm{P}$. Bradshaw, "The turbulence structure of equilibrium boundary layers," Journal of Fluid Mechanics 29, 625 (1967).

${ }^{59}$ P. R. Spalart and J. H. Watmuff, "Experimental and numerical study of a turbulent boundary layer with pressure gradients," Journal of Fluid Mechanics 249, 337 (1993).

${ }^{60}$ S. B. Pope, Turbulent Flows (Cambridge University Press, 2000).

${ }^{61}$ D. Wilcox, Turbulence modeling for CFD (DCW Industries, La Cañada, Calif, 2006).

${ }^{62}$ R. Michel, C. Quémard, and R. Durant, “Application d'un schéma de longueur de mélange à 
l'étude des couches limites turbulentes d'équilibre," Tech. Rep. (ONERA, 1969).

${ }^{63}$ P. S. Klebanoff and Z. W. Diehl, "Some features of artificially thickened fully developed turbulent boundary layers with zero pressure gradient," Tech. Rep. (NACA-TR-1110, National Advisory Committee for Aeronautics, 1952).

${ }^{64}$ P. Spalart and S. Allmaras, “A one-equation turbulence model for aerodynamic flows," La Recherche Aérospatiale (1994).

${ }^{65}$ F. R. Menter, “Two-equation eddy-viscosity turbulence models for engineering applications," AIAA Journal 32, 1598-1605 (1994).

${ }^{66}$ J. Piquet, Turbulent Flows (Springer Berlin Heidelberg, 1999).

${ }^{67}$ P. J. A. Priyadarshana and J. C. Klewicki, "Study of the motions contributing to the Reynolds stress in high and low Reynolds number turbulent boundary layers," Physics of Fluids 16, 45864600 (2004).

${ }^{68}$ J. Klewicki, P. Fife, T. Wei, and P. McMurtry, "A physical model of the turbulent boundary layer consonant with mean momentum balance structure," Philosophical Transactions of the Royal Society A: Mathematical, Physical and Engineering Sciences 365, 823-840 (2007).

${ }^{69}$ A. J. Smits, B. J. McKeon, and I. Marusic, "High Reynolds number wall turbulence," Annual Review of Fluid Mechanics 43, 353-375 (2011), https://doi.org/10.1146/annurev-fluid-122109160753.

${ }^{70}$ N. Hutchins and I. Marusic, "Evidence of very long meandering features in the logarithmic region of turbulent boundary layers," Journal of Fluid Mechanics 579, 1 (2007).

${ }^{71}$ N. Hutchins and I. Marusic, "Large-scale influences in near-wall turbulence," Philosophical Transactions of the Royal Society A: Mathematical, Physical and Engineering Sciences 365, 647-664 (2007).

${ }^{72}$ I. Marusic, R. Mathis, and N. Hutchins, "High Reynolds number effects in wall turbulence," International Journal of Heat and Fluid Flow 31, 418-428 (2010).

${ }^{73}$ J. Cousteix, Turbulence et couche limite (Cepadues-éditions, Toulouse, 1989).

${ }^{74} \mathrm{Z}$. Harun, "The structure of adverse and favourable pressure gradient turbulent boundary layers," (2012). 











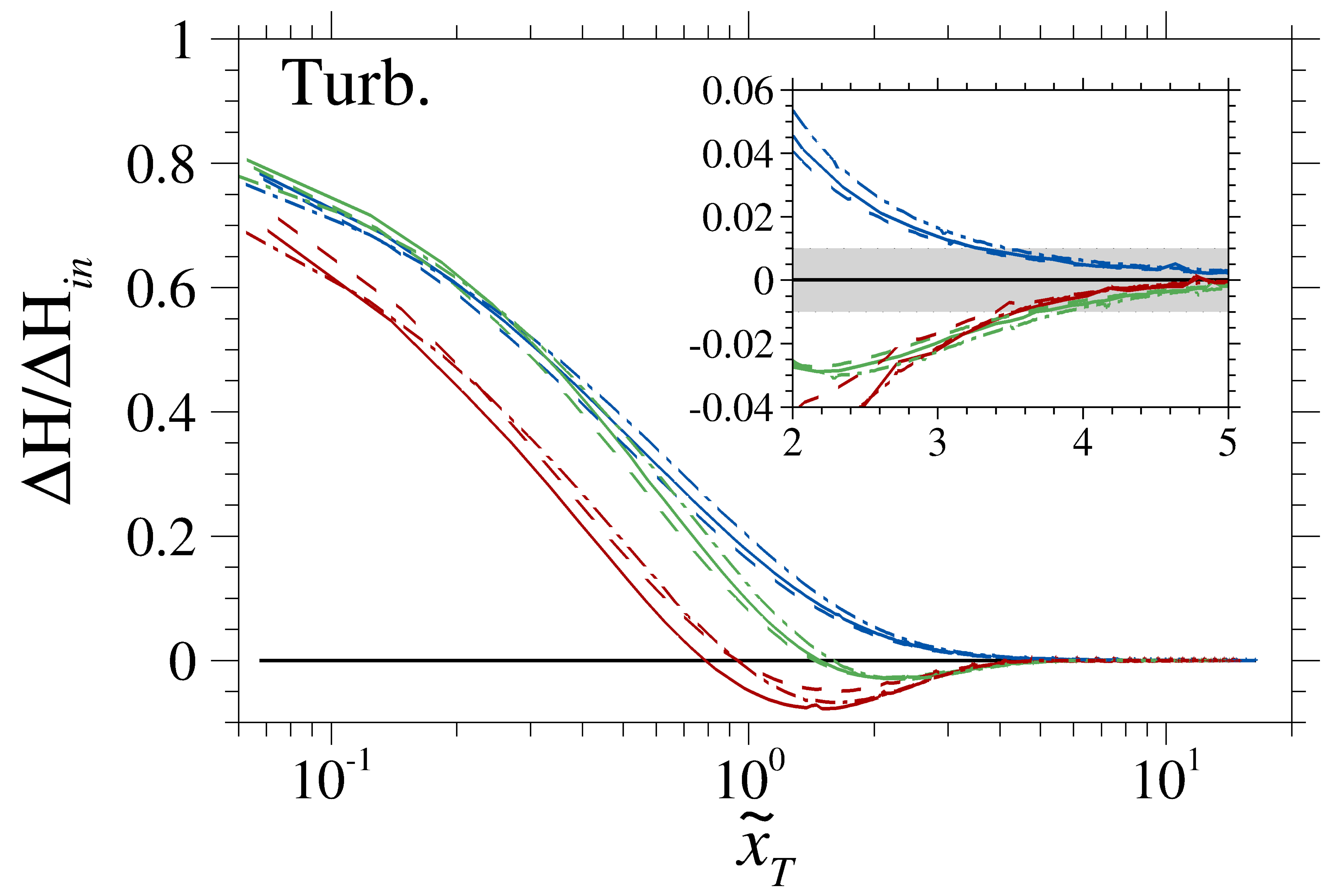




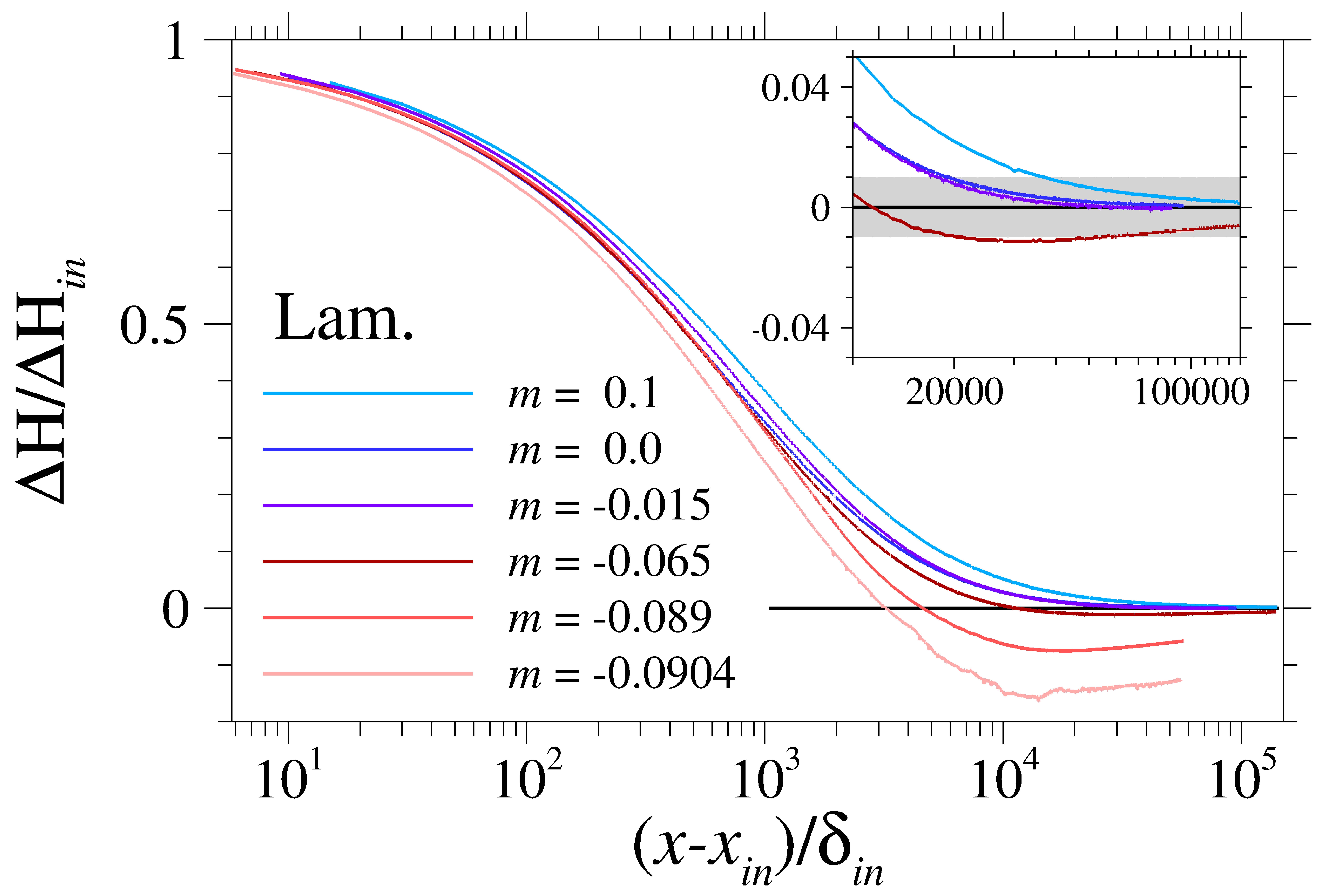






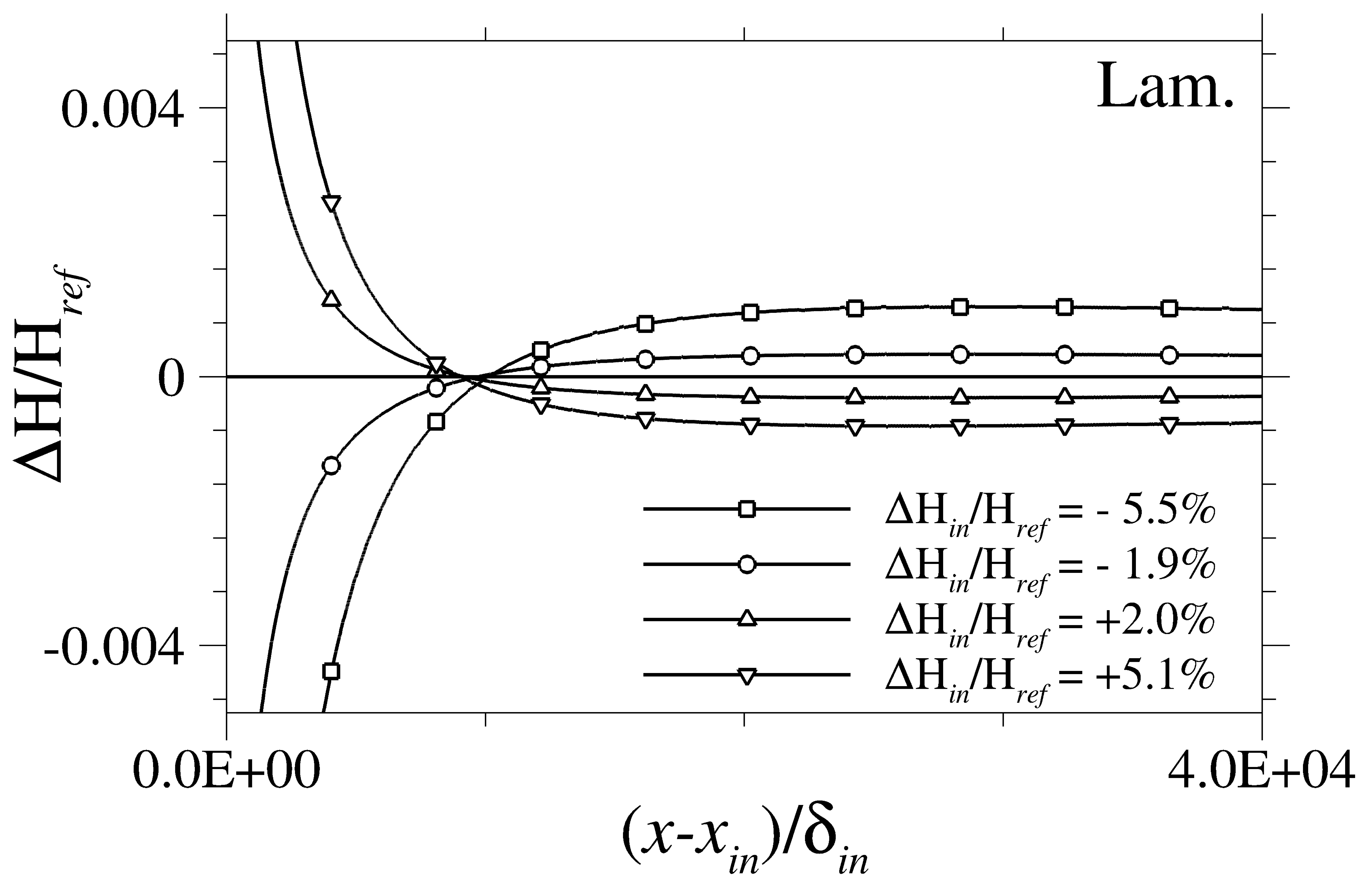




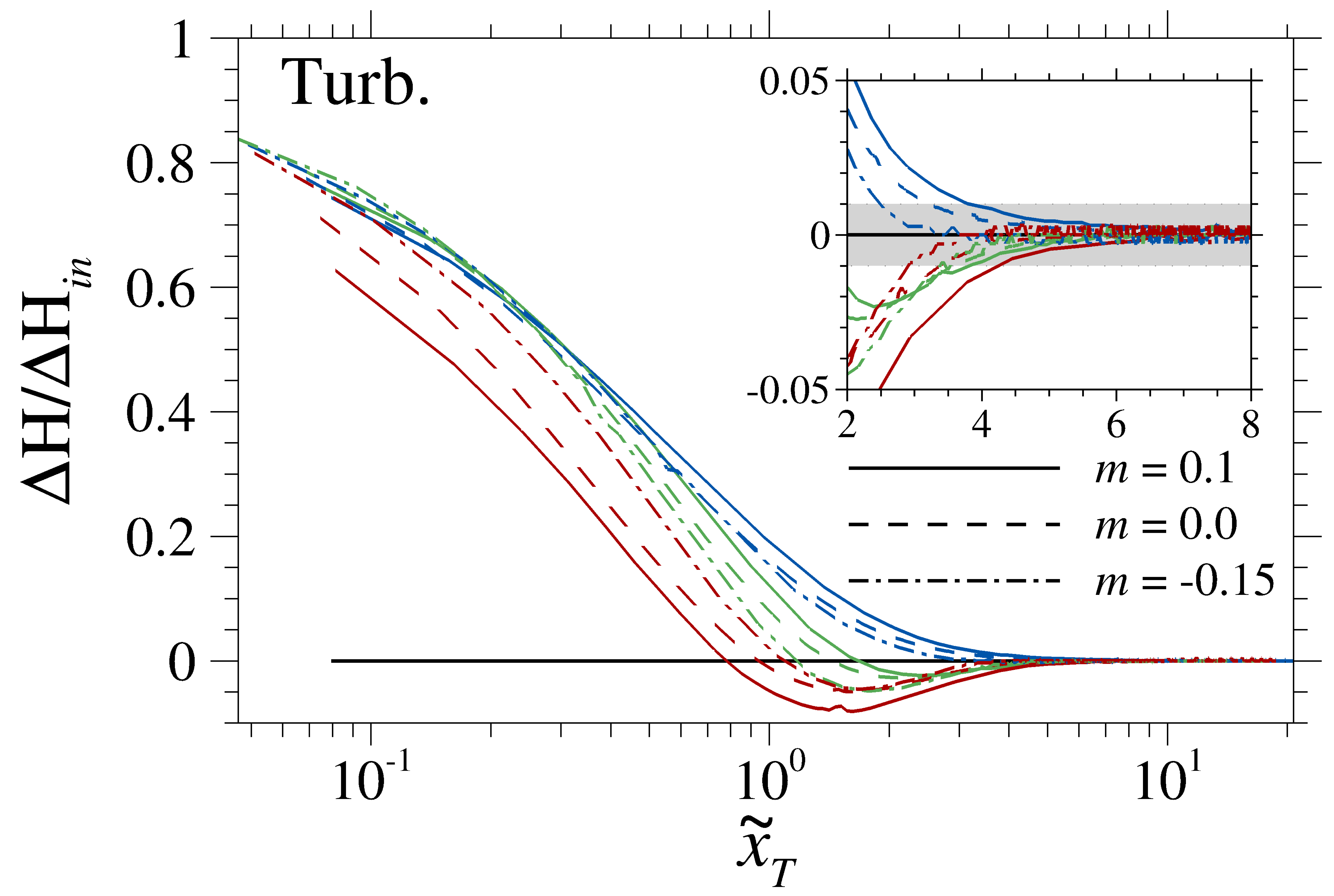




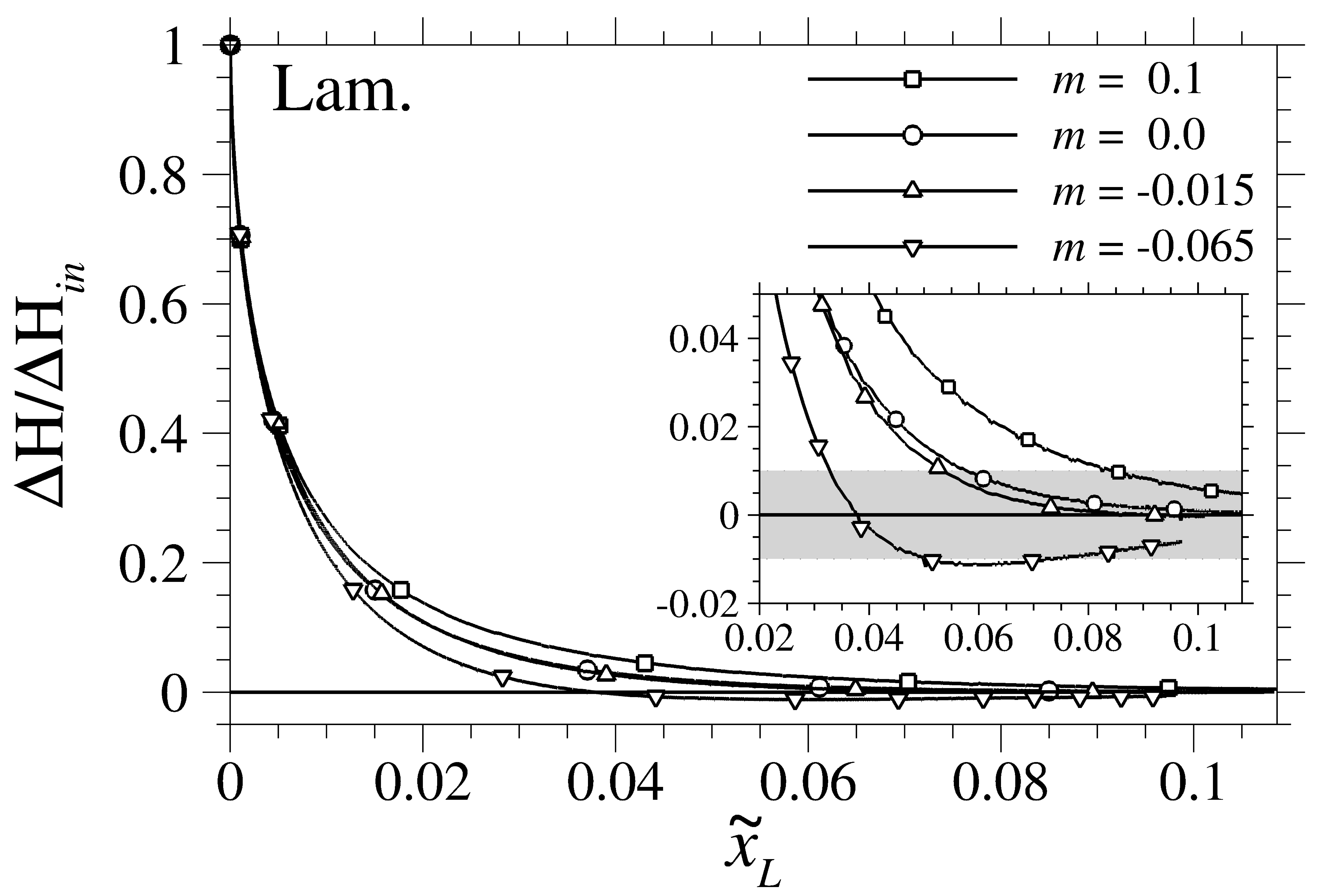




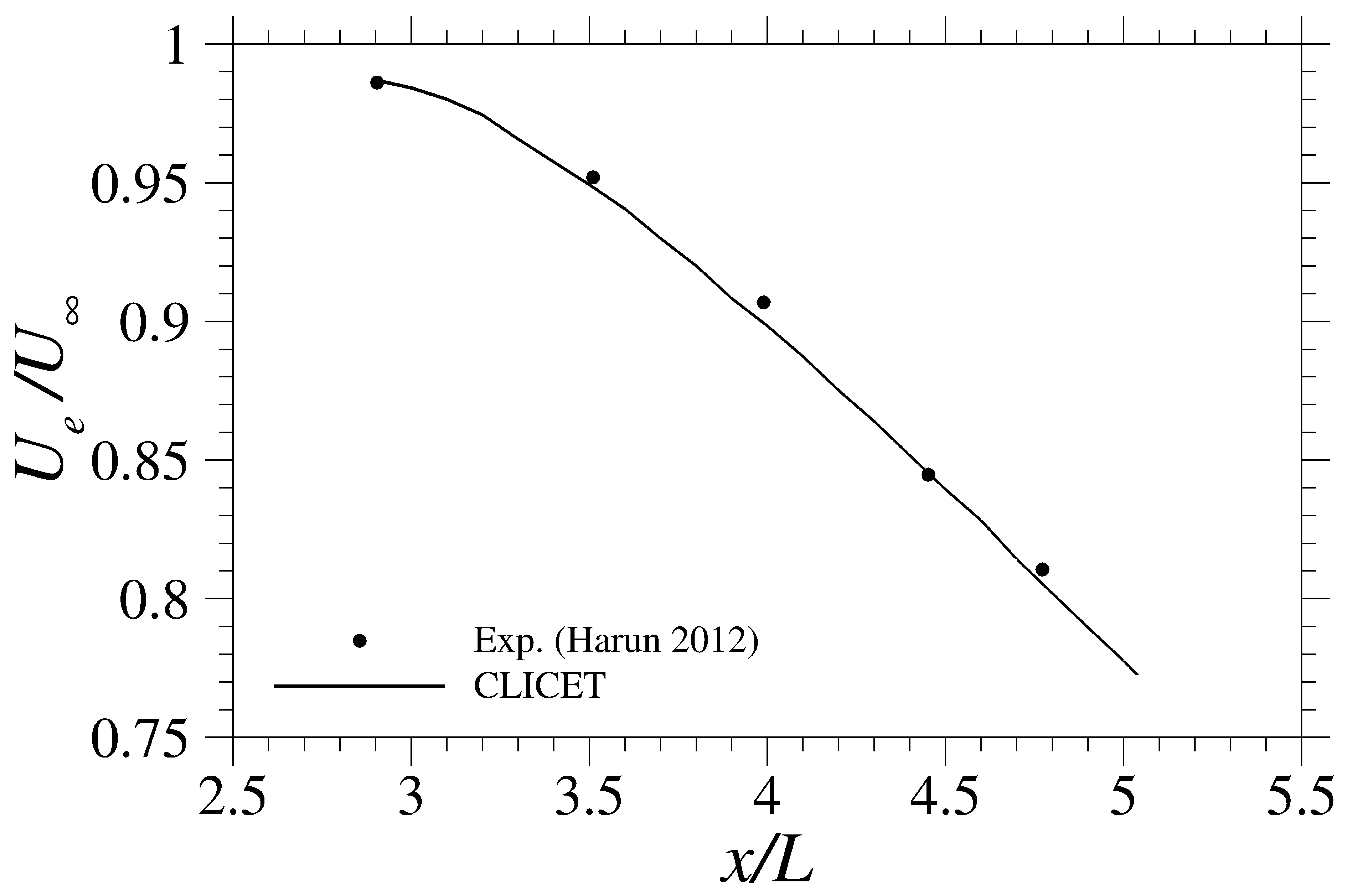




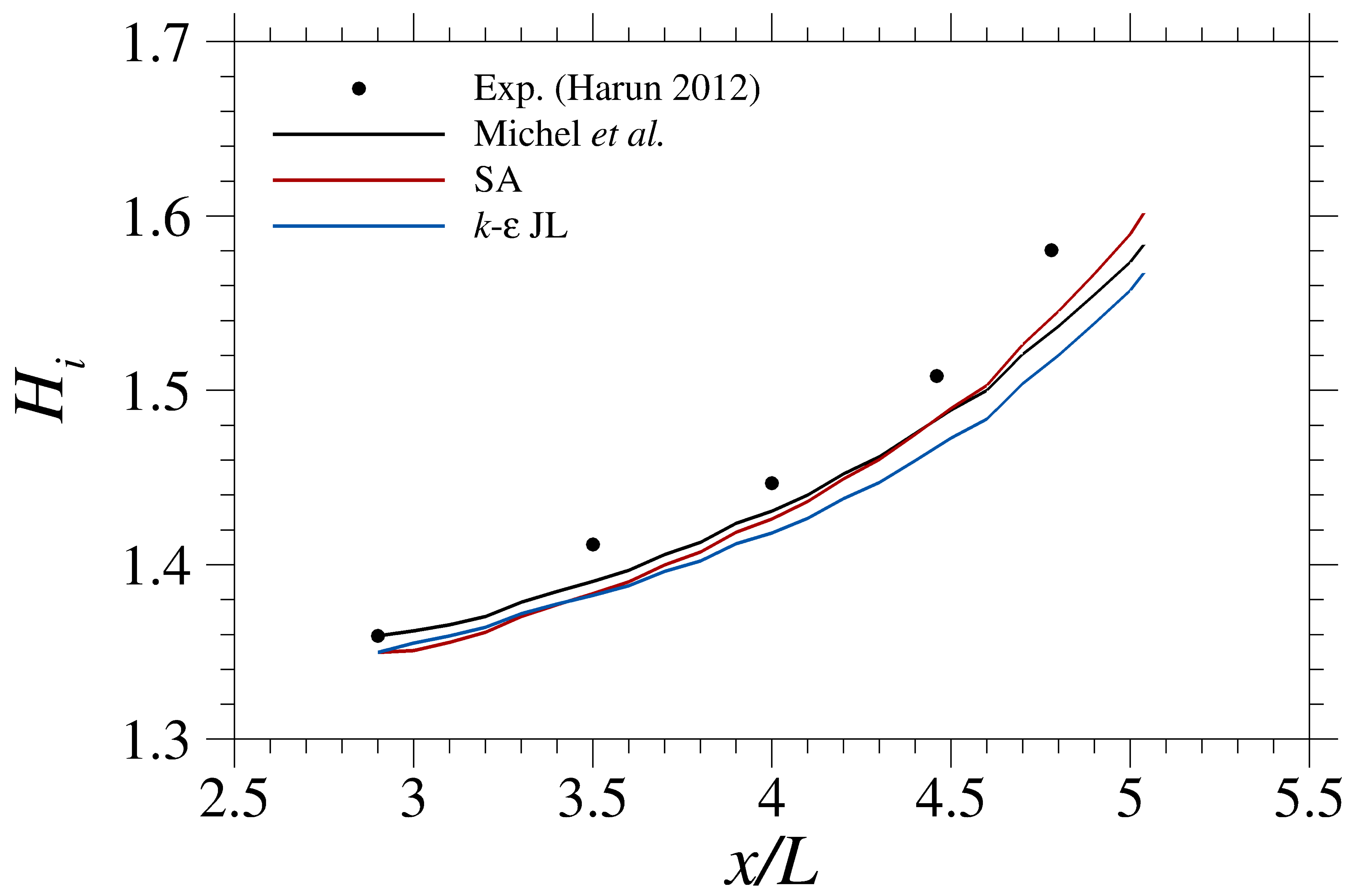

